\title{
47478
}

to
17528

to
17528

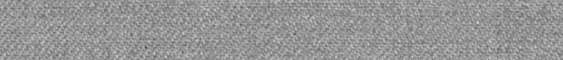

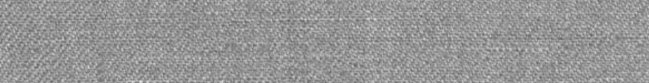

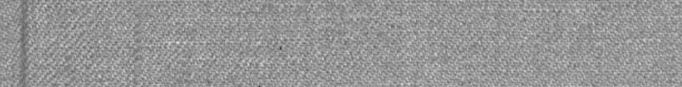

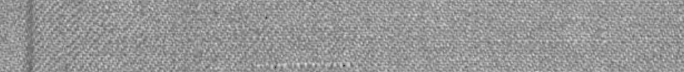

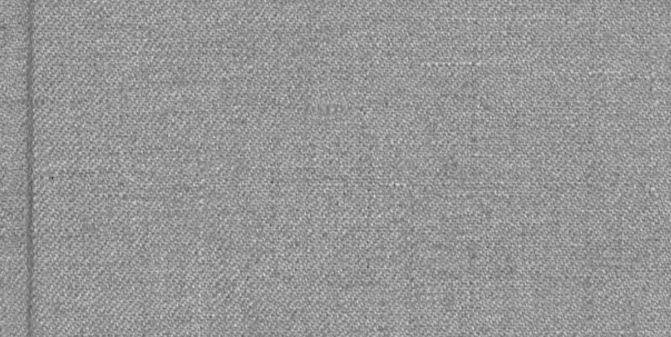

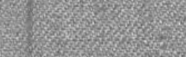

(2)

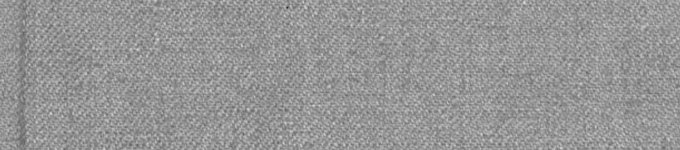

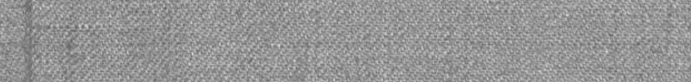
W.

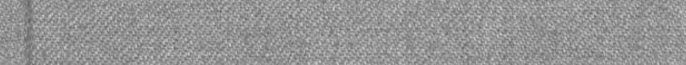
W

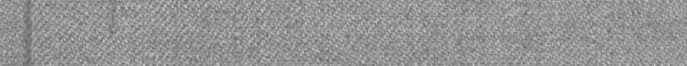

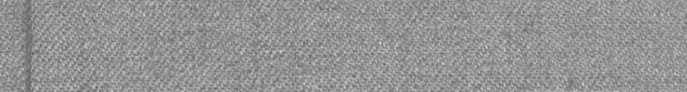
th

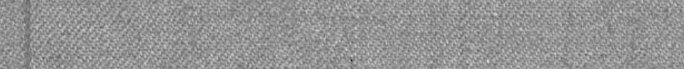

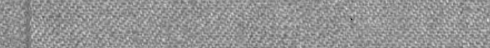

(3)

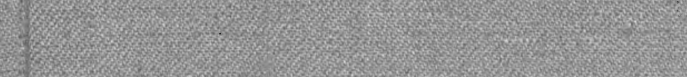
(2)

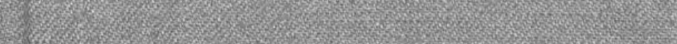

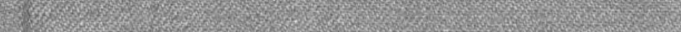

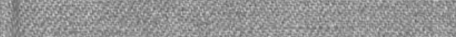


NETS, ETC., USED

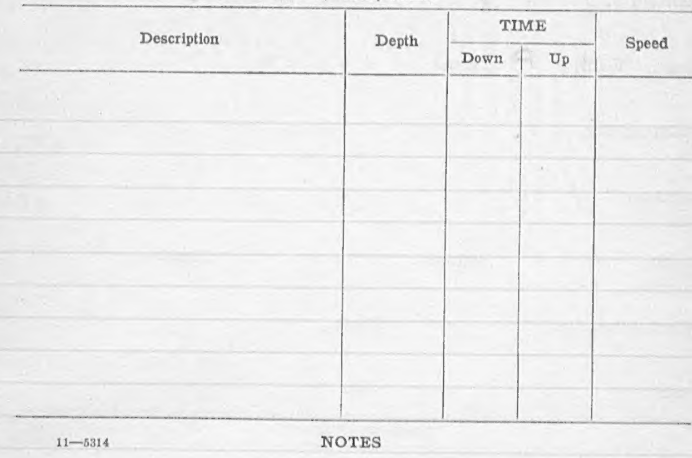


Station No. $F / 479$

Date: 0.7 .17

1916

Hour: $7: 00 /$. M. to

. M.

Position: Lat. $30^{\circ} 30^{\prime} / 5^{\prime \prime} N$ Long. $117^{\circ} 30^{\prime} 0^{\prime \prime} \mathrm{W}$ thut ${ }^{*} \mathrm{l}$ chant $3000 \mathrm{H} .0$.

Iocality:

Depth:

Bottom:

Sample:

Wind: Direction,

Force,

Sea:

Sky:

TEMPERATURES

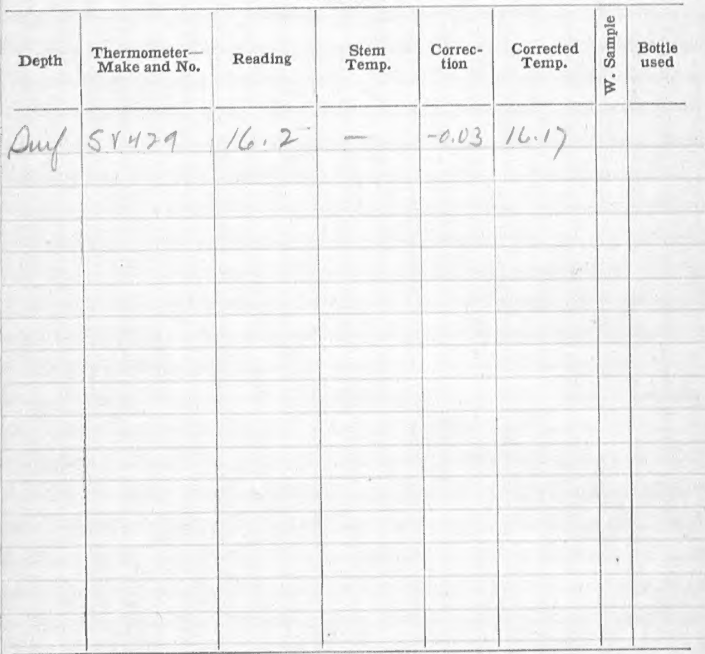

CURRENTS

\begin{tabular}{l|l|l|l|l|l|l}
\hline Depth & Hour & Tide & Time down & Revolutions & Speed & Direction \\
\hline & & & & & & \\
\hline & & & & & & \\
& & & & & & \\
\end{tabular}

Color of water:

Transparency:

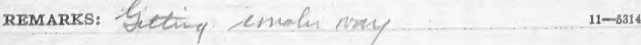


NETS, ETC., USED

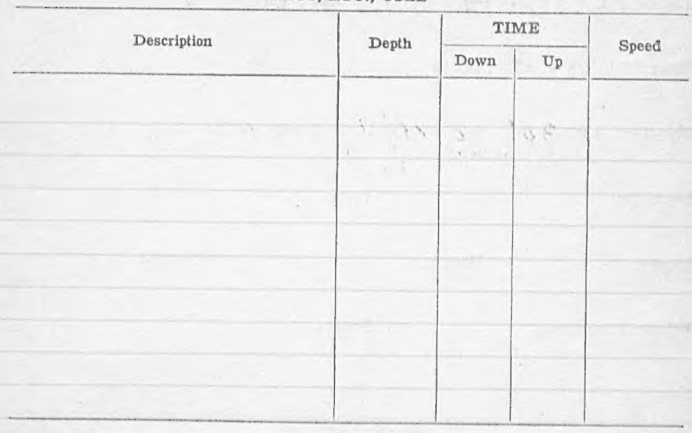

$11-5314$ NOTES 
Station No. $4>480$

Date: 027,17

1916

Hour: $7: 30$ A.M. to . M.

Position: Lat. $30^{\circ} 30^{\prime} 40^{\prime \prime} \mathrm{N}$ Long. $117^{\circ} 31^{\prime} 0^{\prime \prime} \mathrm{W}$ Dhutic chant $3000 \% .0$

Locality:

Depth:

Bottom:

Sample:

Wind: Direction,

Force,

Sea:

Sky:

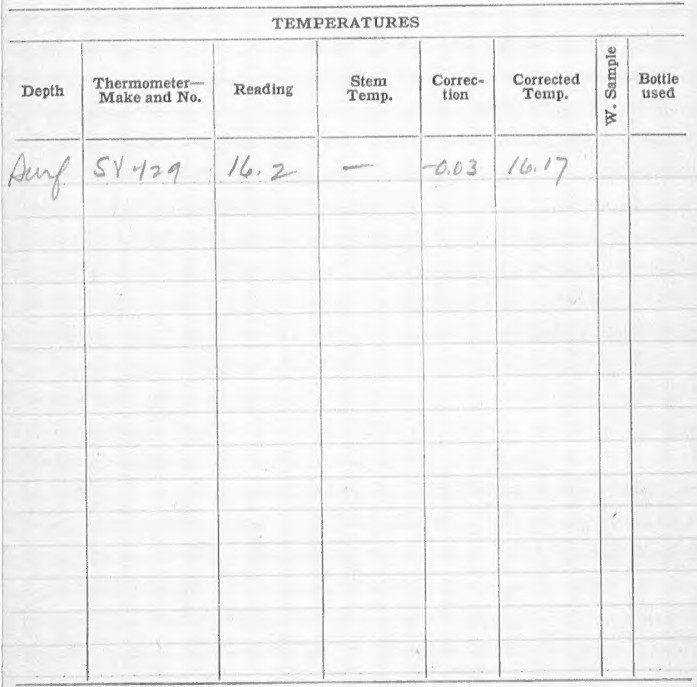

CURRENTS

\begin{tabular}{l|l|l|l|l|l|l}
\hline Depth & Hour & Tide & Time down & Revolutions & Speed & Direction \\
\hline & & & & & \\
\hline & & & & & \\
& & & & & \\
& & & & & & \\
\end{tabular}


NETS, ETC., USED

\begin{tabular}{l|l|l|l|l|}
\hline Description & Depth & \multicolumn{2}{|c|}{ TIME } & Speed \\
\cline { 2 - 4 } & Down & Up & \\
\hline & & & \\
& & & \\
& & & \\
\end{tabular}


Station No. 47481

Hour: $8: 00 \neq$. M. to

Date:

1916

Position: Lat. $30^{\circ} 31^{\prime} 20^{\prime \prime} \mathrm{N}$ Long. $117^{\circ} 33^{\prime} 25^{\prime \prime} \mathrm{W}$ Dhut ${ }^{2} \mathrm{C}$ chat $3000 \mathrm{H} .0$.

Locality:

Depth:

Bottom:

Sample:

Wind: Direction,

Force,

Sea:

Sky:

TEMPERATURES

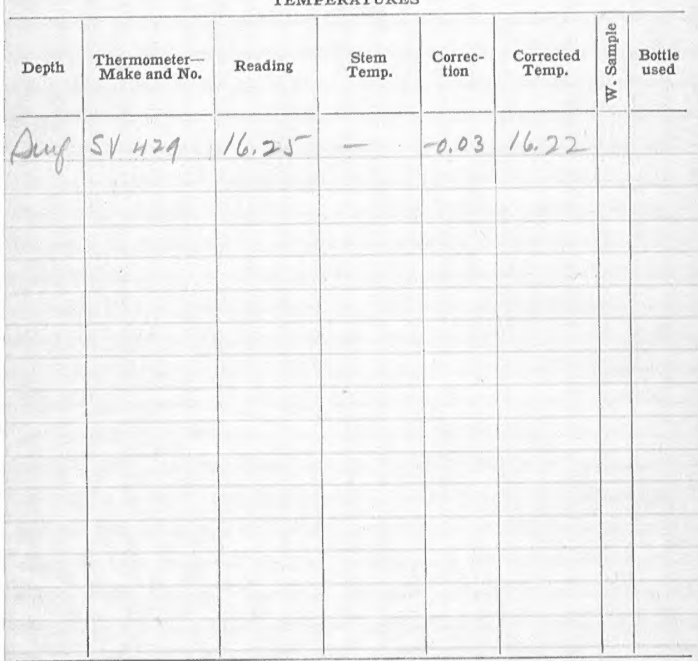

CURRENTS

\begin{tabular}{l|l|l|l|l|l|l}
\hline Depth & Hour & Tide & Time down & Revolutions & Speed & Direction \\
\hline & & & & & \\
\hline & & & & & \\
& & & & & & \\
& & & & &
\end{tabular}

Transparency:

REMARKS: doweln pray 
NETS, ETC., USED

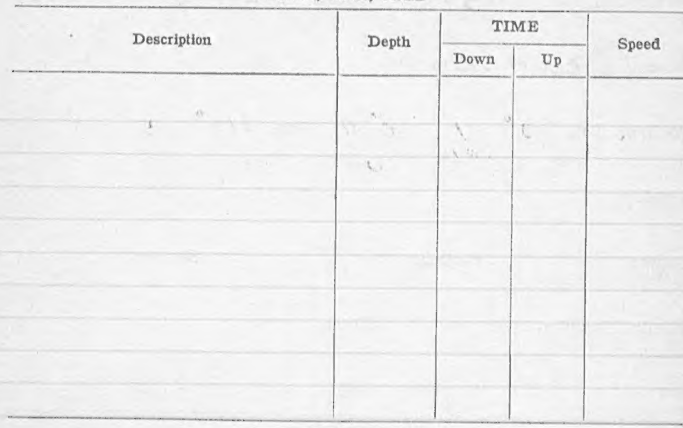




Station No. 17482 Date: 0.1916

Hour: $8,30 A . M$. to . M.

Position: Lat. $30^{\circ} 32^{\prime} 0^{\prime \prime} \mathrm{N}$ Long. $117^{\circ} 36^{\prime} 0^{\prime \prime} \mathrm{W}$ Ohut 6 chant $3000 \% 0$.

Locality:

Depth:

Bottom:

Sample:

Wind: Direction,

Sea:

Sky:

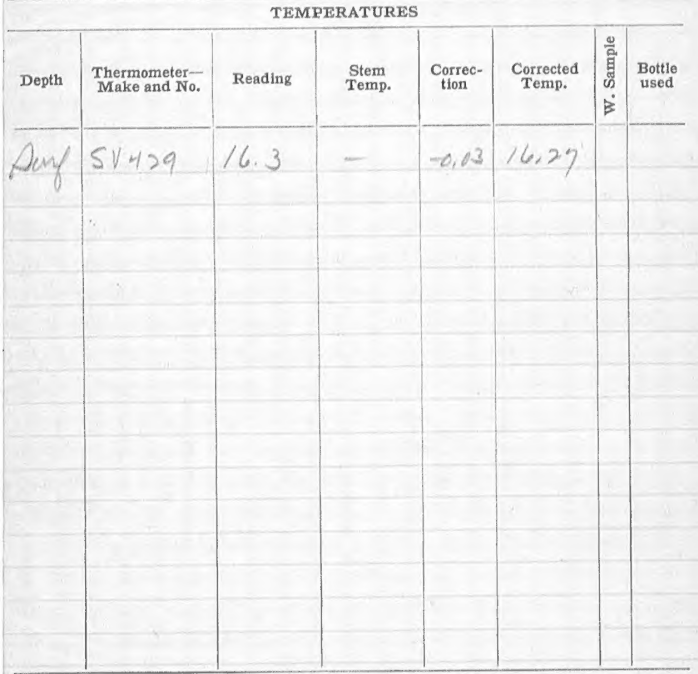

CURRENTS

\begin{tabular}{l|l|l|l|l|l|l}
\hline Depth & Hour & Tide & Time down & Revolutions & Speed & Direction \\
\hline & & & & & \\
\hline & & & & & \\
& & & & & & \\
& & & & & & \\
\end{tabular}

Transparency:

REMARKS:

Lluaber rey

Force, 
NETS, ETC., USED

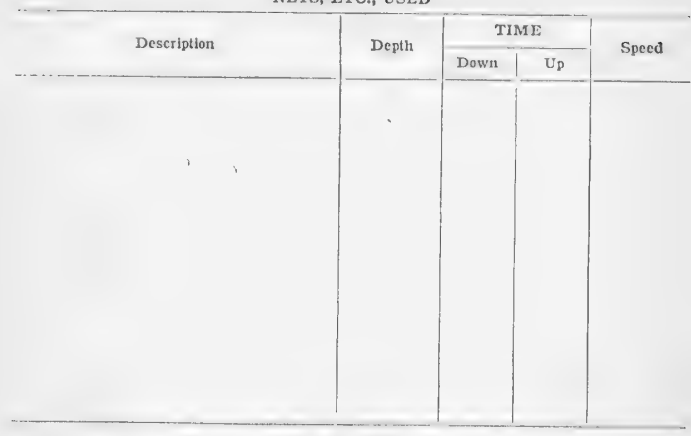


Station No. $\%, \cdots, 3$

Date:,,$- ;$

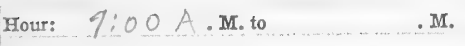

Position: Lat. $30^{\circ} 32^{\prime} 45^{\prime \prime} \mathrm{N}$ Long. $117^{\prime \prime} 38^{\prime} 55^{\circ} \mathrm{h}$

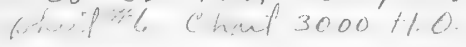

Locality:

Depth:

Bottom:

Sample:

Wind: Direction,

Force,

Sea:

Sky:

TEMPERATURES

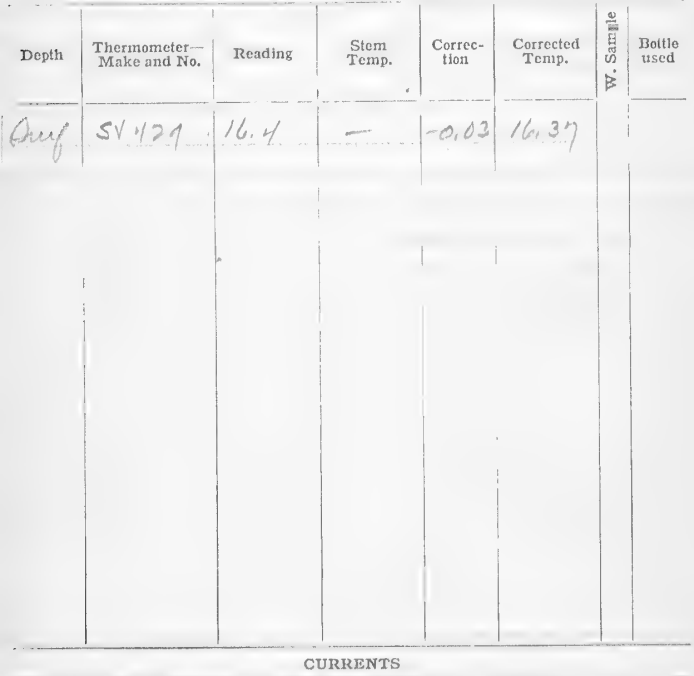

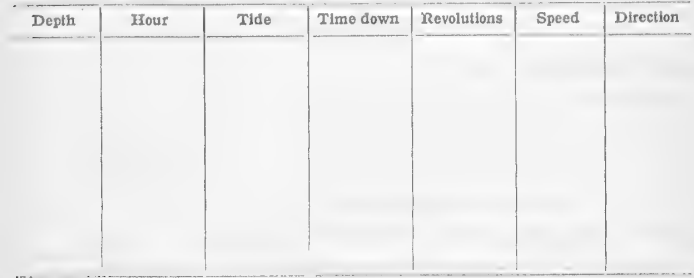


NETS, ETC., USED

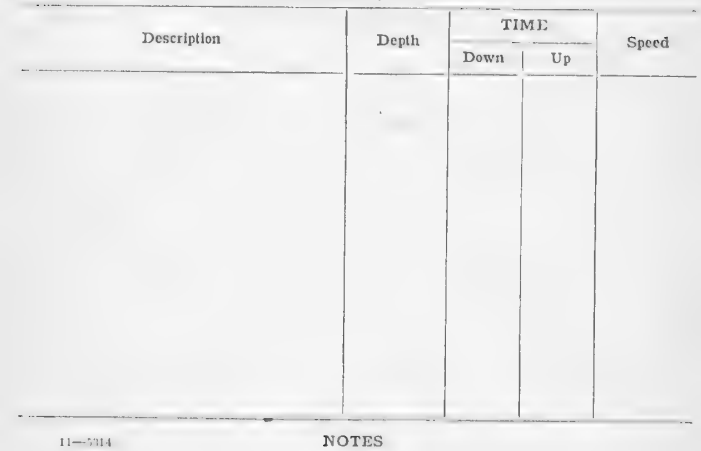


NETS, ETC., USED

Description $\mid$


Station Iro.

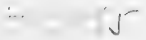

Date:

i $\quad \dot{i}$

191

Hour: $\quad 10 ; 00$ A. M. to

. M.

Position: Lat. $30^{\circ} 34^{\prime} / S^{\prime \prime} N$ Long. $117^{\circ}, 14^{\prime}$, I N Ohut 5 Chat $3000 \mathrm{t} .0$.

Locality:

Depth:

Bottom:

Sample:

Wind: Direction,

Force,

Sea:

Sky:

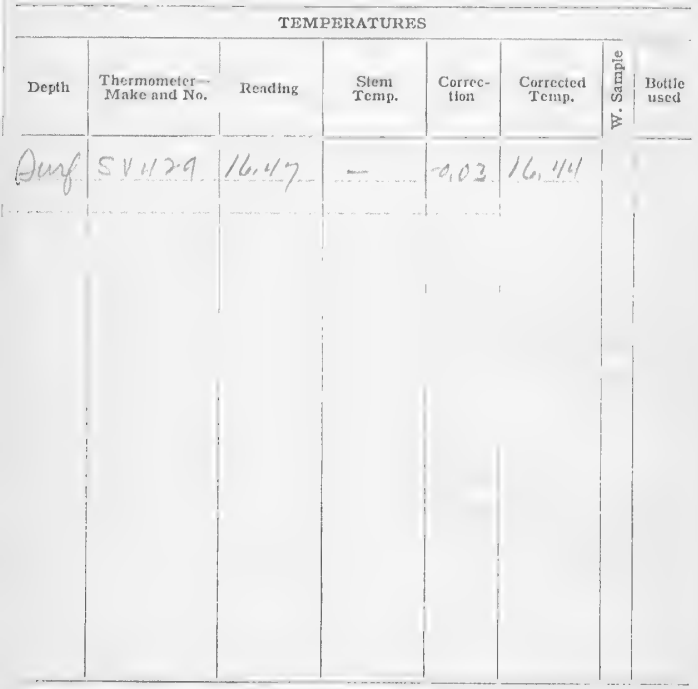

CURRENTS

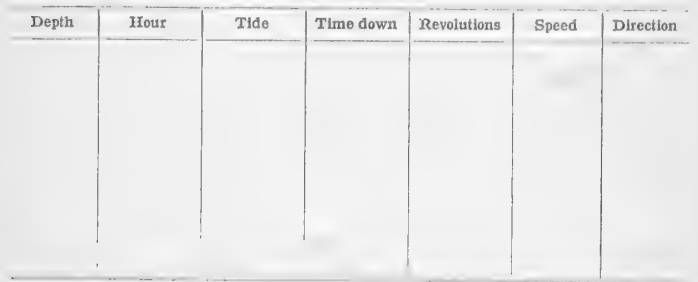

Color of water:

Transparency:

REMARKS:

Loveriver

11- 
NETS, ETC., USED

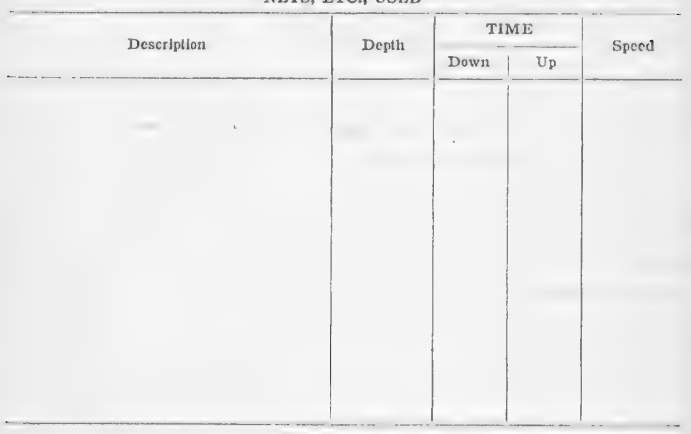

$11-5: 314$ NOTES 
Locality:

Depth:

Bottom:

Sample:

\section{Wind: Direction,}

Sea:
Sky:

\section{Force,}

TEMPERATURES

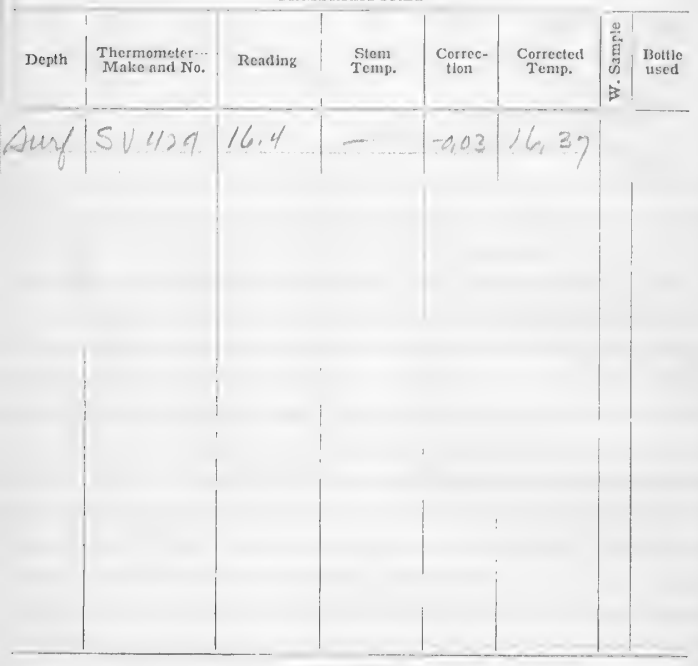

CURRENTS

\begin{tabular}{l|l|l|l|l|l|l}
\hline Depth & Tour & Time down & Revolutions & Speed & Direction \\
\hline & & & & & & \\
& & & & & & \\
& & & & & &
\end{tabular}


NETS, ITC., USED

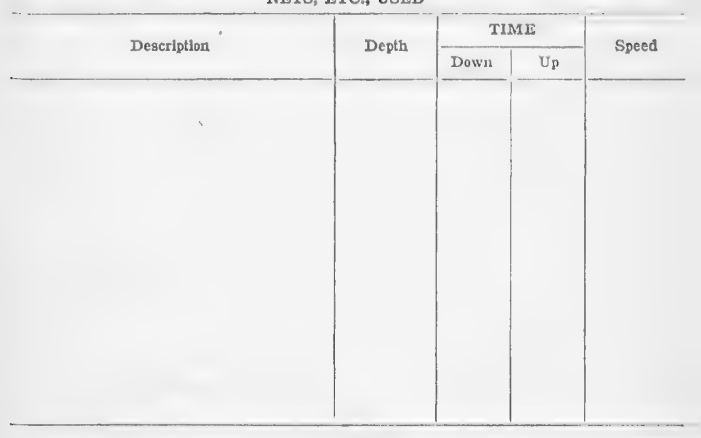

$11-5314$

NOTES 
Station No. HF, :

Date:

191 〈.

Hour: 1/:00 4 . to . . M.

Position: Lat. $30^{\circ} 35^{\prime} 30^{\prime \prime} N$ Long. $117^{\circ} 50^{\prime} 0^{\prime \prime} 1$, $A \therefore ; 706$ Chout $3000+100$

Iocality:
Depth:
Bottom:
Sample:

Wind: Direction,

Force,

Sea:

Sky:

TEMPERATURES

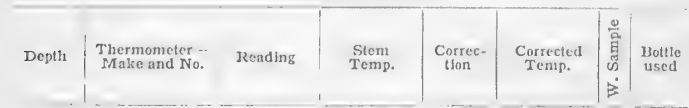

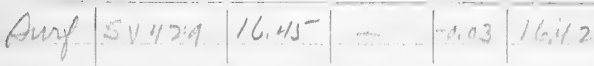

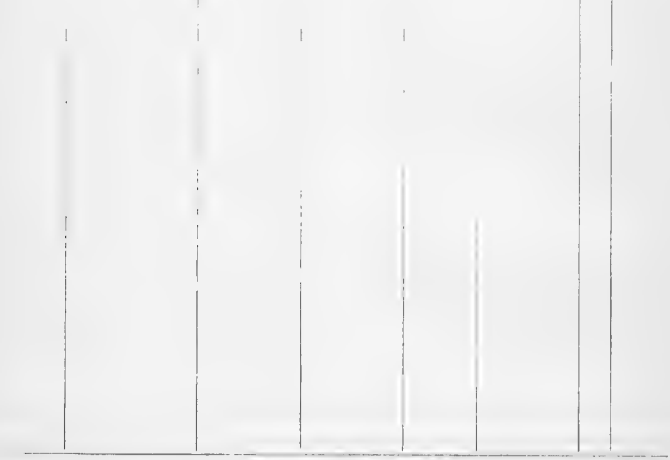

\begin{tabular}{|c|c|c|c|c|c|c|}
\hline \multicolumn{7}{|c|}{ CURRENTS } \\
\hline Depth & Hour & Tide & Time down & Revolutions & Speed & Direction \\
\hline & & & & & & \\
\hline & & & & & & \\
\hline & & & & & & \\
\hline & & & & & & \\
\hline & & & & & & \\
\hline & & & & & & \\
\hline
\end{tabular}


NETS, ETC., USED

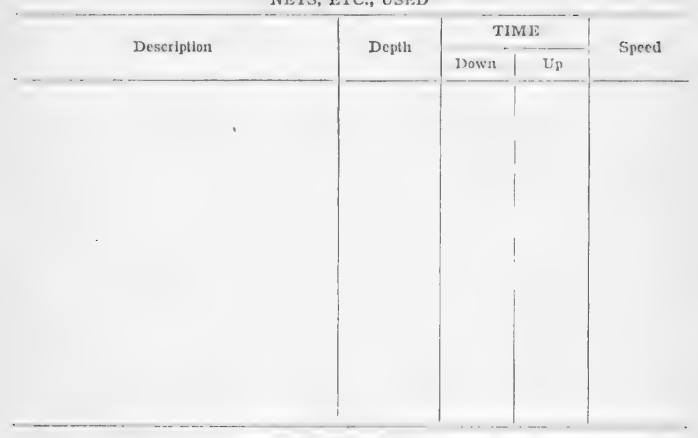




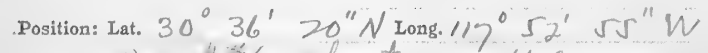
$\therefore$ ine, is, hat $3000 \mathrm{Ht}$.

\section{Locality:}

Depth:

Wind: Direction,

Sea:
Bottom:

Force,

Sky:

TEMPERATURES

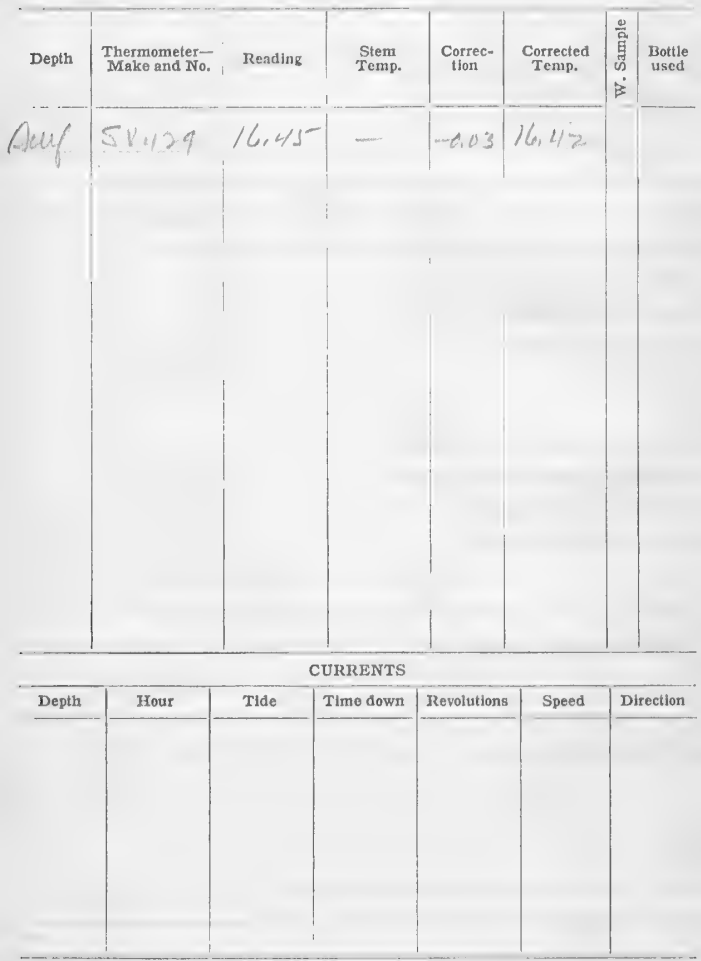


NETS, FTC., USED

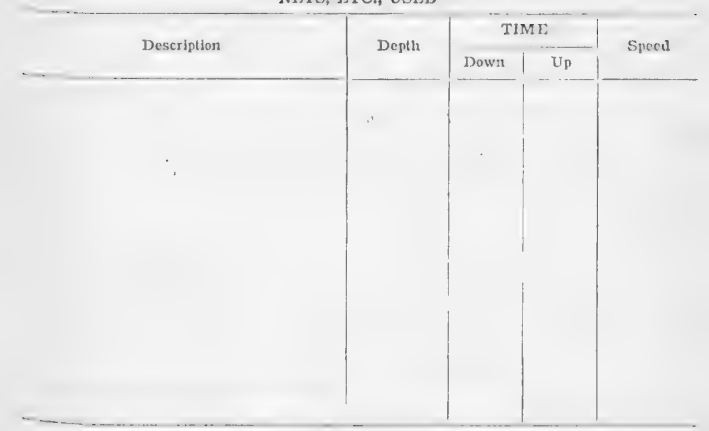

11- -ia1!

NOTES 
Station No. $t / ; i q$

Date:

$c^{\prime},+i, j$

$191 \zeta$

Hour: $12: 00$. M. to

. M.

Position: Lat. $30^{\circ} 37^{\prime} / 5^{\prime \prime} N$ Long. $117^{\circ} 5^{\prime} 3 J^{\prime} \mathrm{VV}$ Phutt: chant $3000 \mathrm{tt} 0$

Locality:

Depth:

Bottom:

Sample:

Wind: Direction,

Sea:

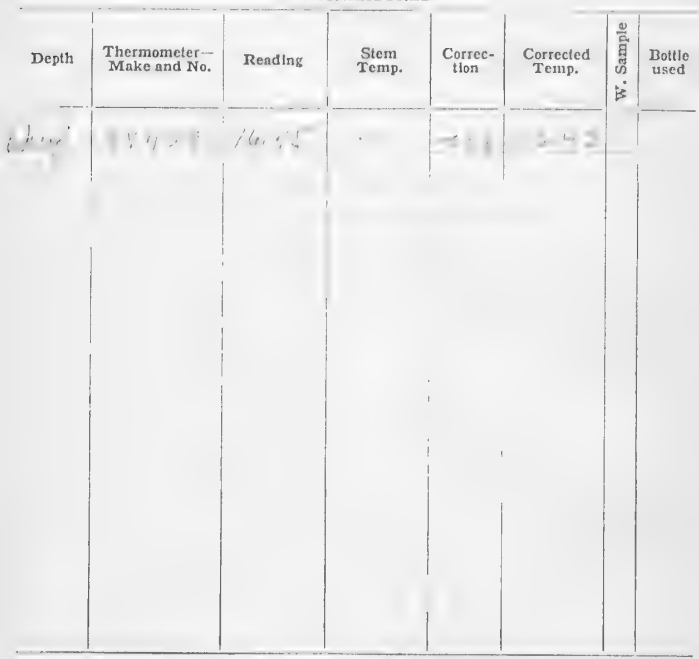

CURRENTS

\begin{tabular}{l|l|l|l|l|l|l}
\hline Depth & Ilour & Tide & Time down & Revolutions & Speed & Direction \\
\hline & & & & & \\
& & & & & \\
& & & & & & \\
& & & & & & \\
\end{tabular}

Color of water:

Transparency:

REMARKS

Limber may

Force,

Sky: 
NETS, ETC., USED

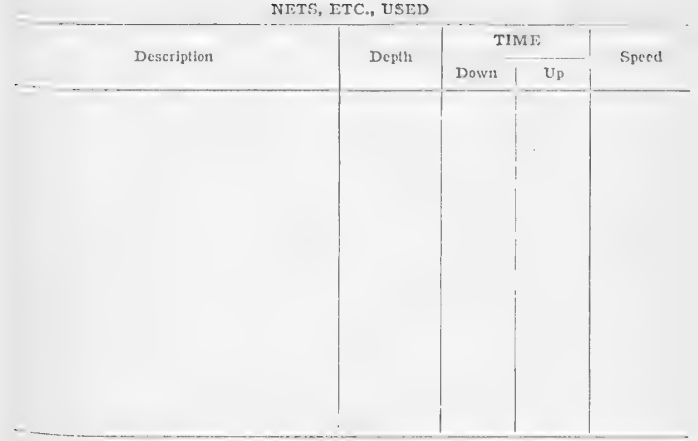


NITS, ITC., USIED

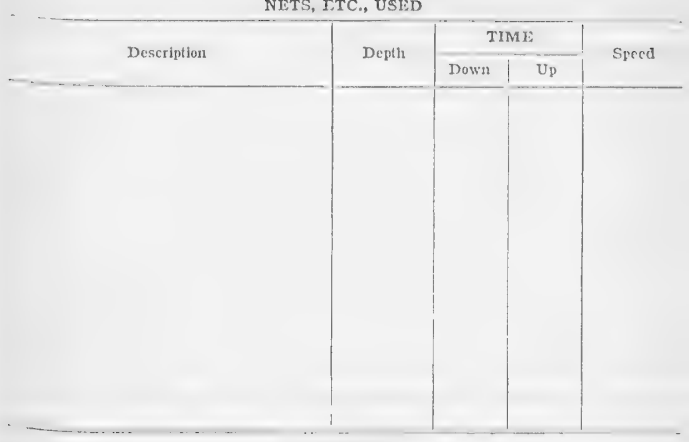

NOTES 
Station No. $\mathrm{H}>4 \mathrm{A2} \quad \ldots$ Date: Cet, 7916

How: $/ 1,30 P$. M. to . M.

Position: Lat. $30^{\circ} / /^{\prime} \sqrt{5} s^{\prime \prime} N$ Long. $/ / S^{\circ} \gamma^{\prime} 5$ M Sine

Locality:
Depth:
Bottom:
Sample:

Wind: Direction,

Sea:
Force,

Sky:

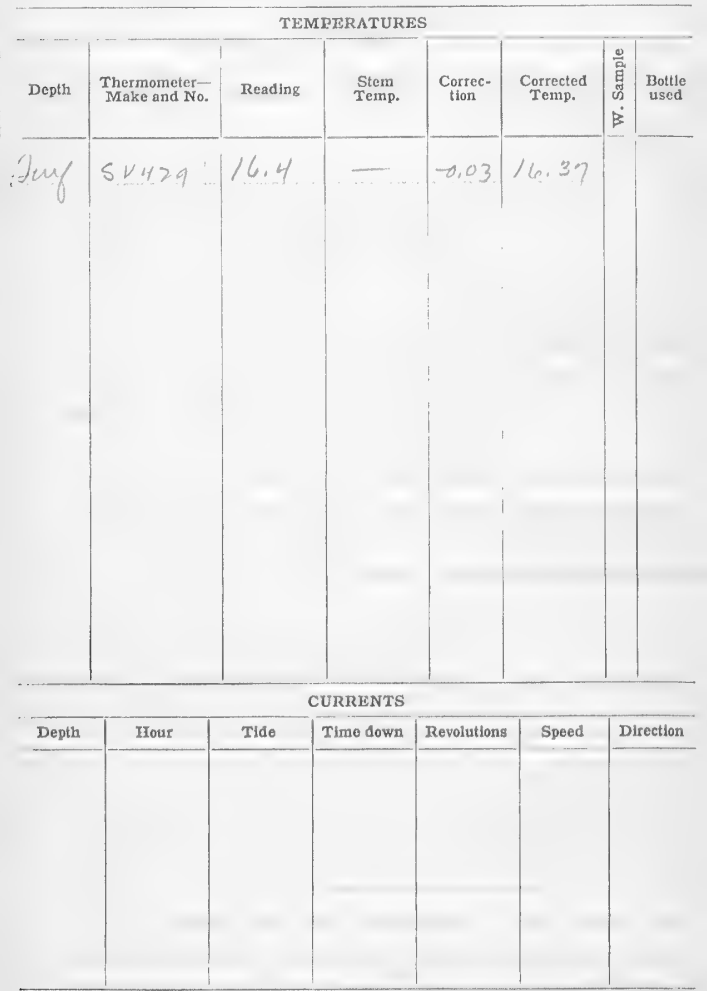


NETS, ITC., USED

Description


NETS, ETC., USED

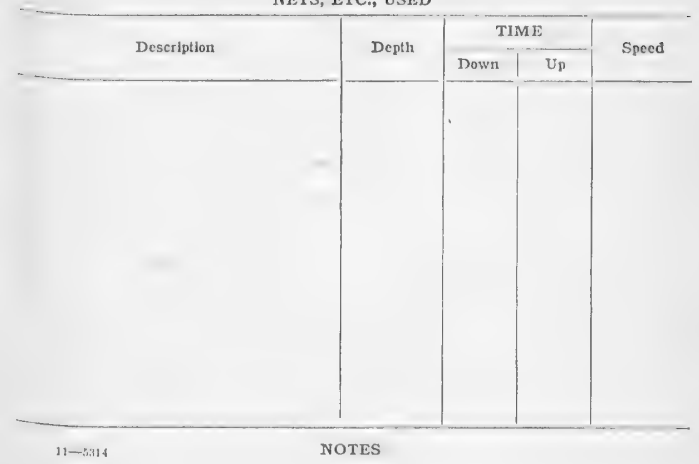


Hour: $2: 30 P$. M. to M.

Position: Lat. $30^{\circ} 4 S^{\prime} / S^{\prime \prime} N$ Long. $1 / \delta^{\circ} S^{1} 30 \cdots$ A) wet "le ant

Locality:

Depth:

Bottom:

Sample:

Wind: Direction,

Force,

Sea:

Sky:

TEMPTRATURES

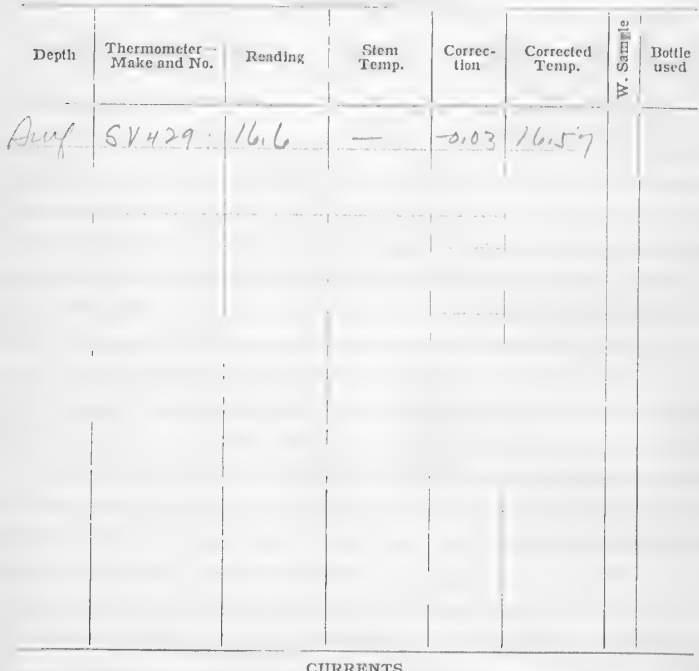

CURRENTS

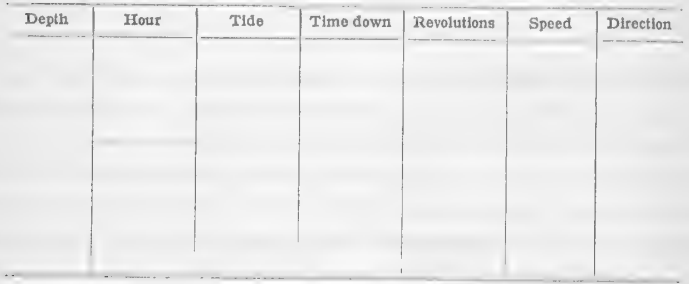

Color of water:

Transparency:

REMARKS:

Muren rray 
NETS, ETC., USED

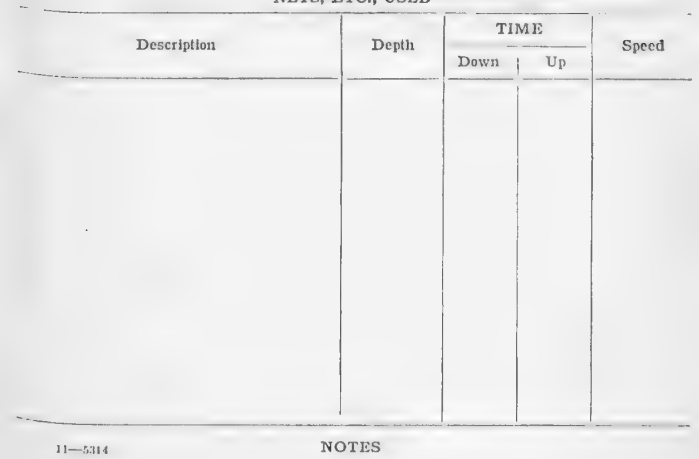


NETS, ETC., USED

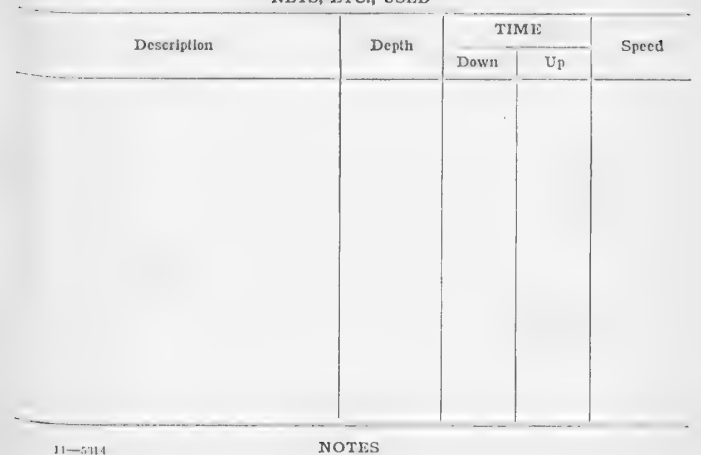


Station No. $\cdots ; \ddot{l l i}$

Hour: $3: 30 P . M$. to . M.

Position: Lat. $30^{\circ} 48^{\prime} 10^{\prime \prime} \mathrm{N}$ Long. $118^{\circ} 8^{\prime} 30^{\prime \prime} \mathrm{W}$

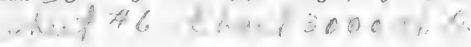

Locality:

Depth:

Bottom:

Sample:

Wind: Direction,

Force,

Sea:

Slsy:

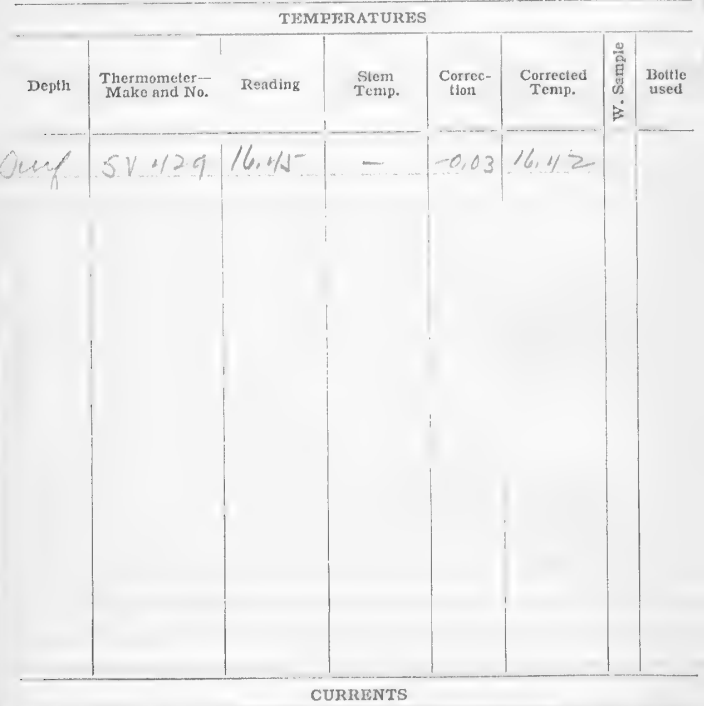

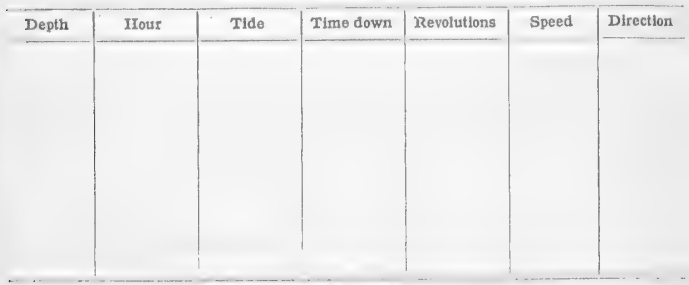

Color of water:

Transparency:

REMARKs: 1 luelu irne, $11-5314$ 
NETS, ETC., USED

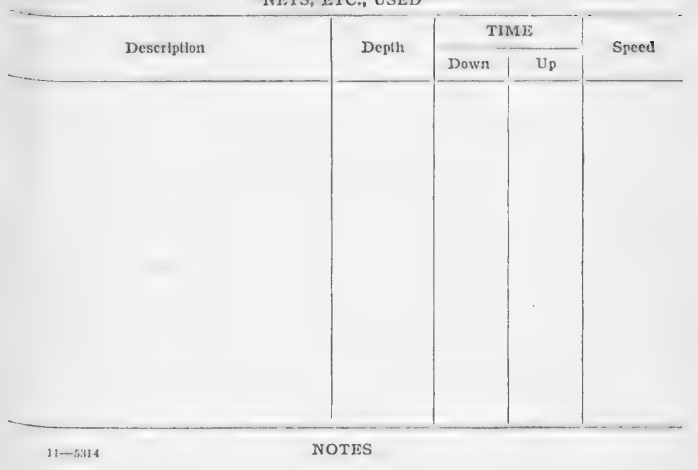


NFTS, ITC, USTD

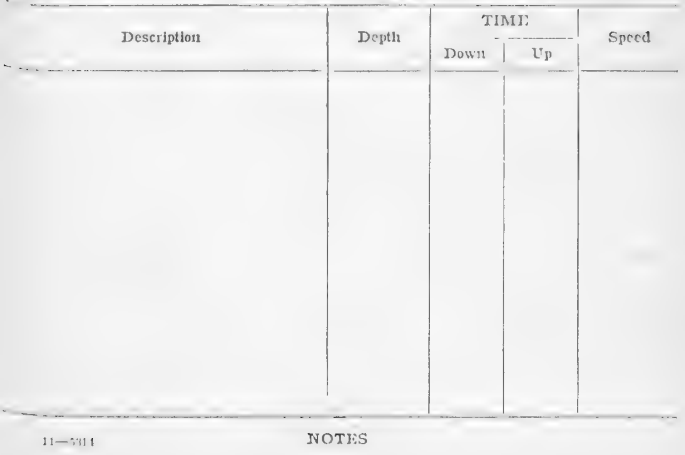


Hour: $7: 00$ A. M. to . M.

Position: Lat. $30^{\circ} 43^{\prime} 10^{\prime \prime} N$ Long. $118^{\circ} 21^{\prime} 1 / 5^{\circ} \mathrm{W}$ thut 6 chat $3000 \mathrm{HLO}$.

Locality:

Depth:

Bottom:

Sample:

Wind: Direction,

Force,

Sea:

Sky:

TEMPERATURES

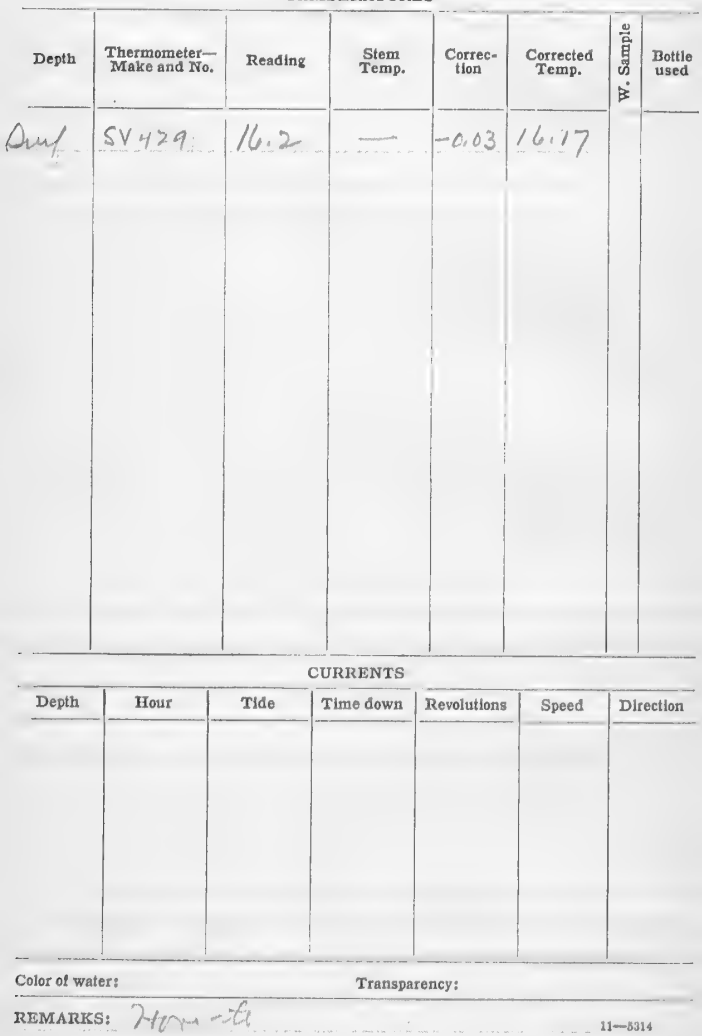


NITS, ETC., USTD

Description


Hour: $7: 30$ A.M. to

. M.

Position: Lat. $30^{\circ} 44^{\prime} 0^{\prime \prime} N$ Long. $118^{\circ} 24^{\prime} 40^{n} \mathrm{~W}$ thut $L$ Chat 3000 . H. O.

Locality:

Depth:

Bottom:

Sample:

Wind: Direction,

Sea:

Sky:
Force,

EMPERATURES

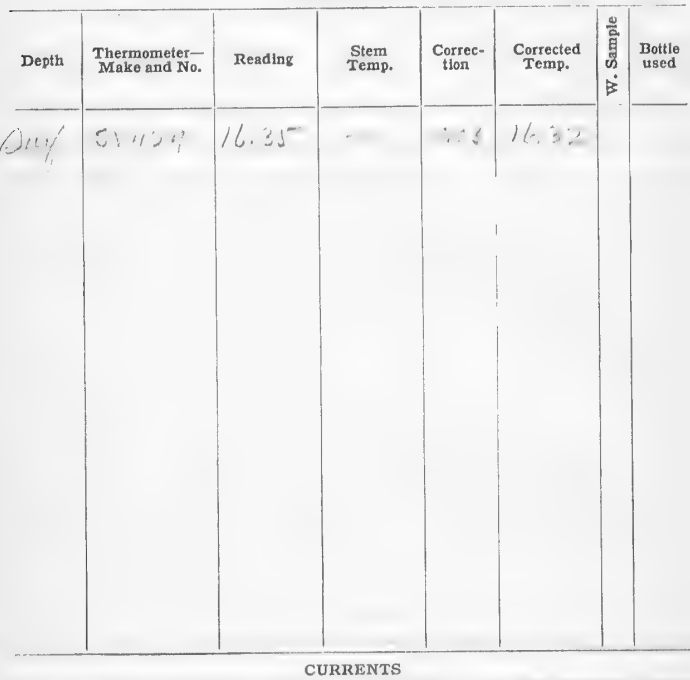

\begin{tabular}{c|c|c|c|c|c|c}
\hline Depth & Ifour & Tide & Time down & Revolutions & Speed & Direction \\
\hline & & & & \\
& & & & & \\
& & & & &
\end{tabular}

Color of water: $11-5314$ 
NETS, ITC., USED

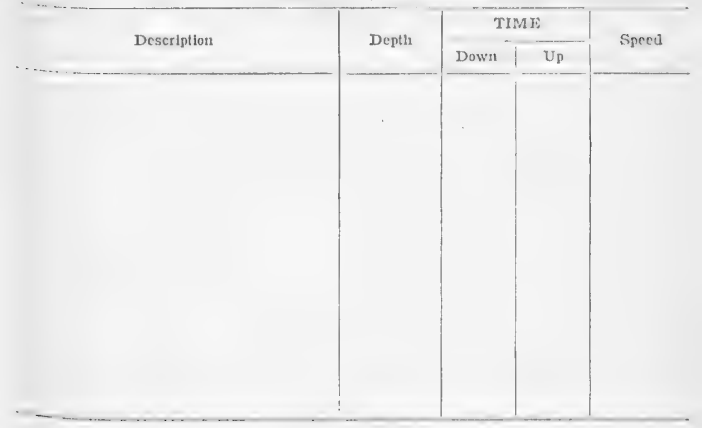


Station No. H\% 1500

Date: $C, i, \cdots 191$

Hour: $8: 00$ A.M. to

. M.

Position: Lat. $30^{\circ} 44^{\prime} 25^{\prime \prime} \mathrm{N}$ Long. $118^{\circ} .7^{\circ} 10^{\circ} \mathrm{iN}$ thuttil chant $3000 \mathrm{H.O}$

Locality:

Depth:

Bottom:

Sample:

Wind: Direction,

Sea:

Sky:

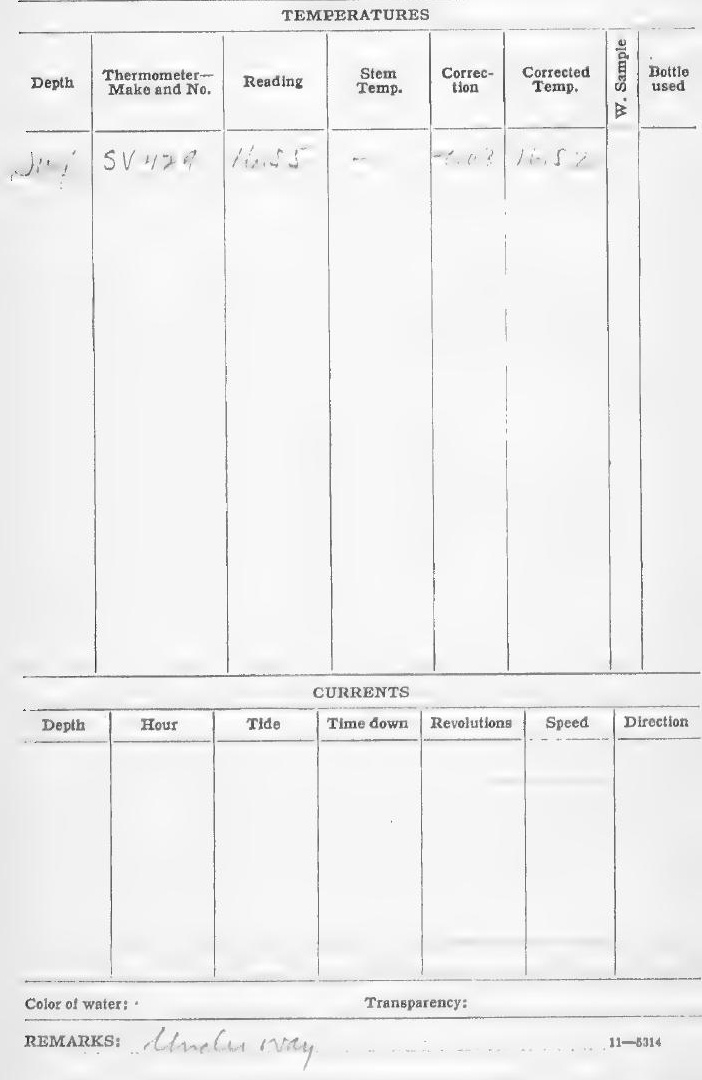


NETS, ETC., USED

Description




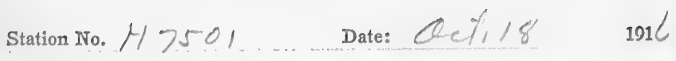
Hour: $8: 30$ A.M. to .M.

Position: Lat. $30^{\circ} 45^{\prime} 10^{\prime \prime} \mathrm{N}$ Long. $118^{\circ} 30^{\prime} 0^{\prime \prime} \mathrm{W}$ thut 6 Chat $3000 \mathrm{M} .0$.

Locality:

Depth:

Bottom:

Sample:

Wind: Direction,

Sea:

TEMPIRATURES

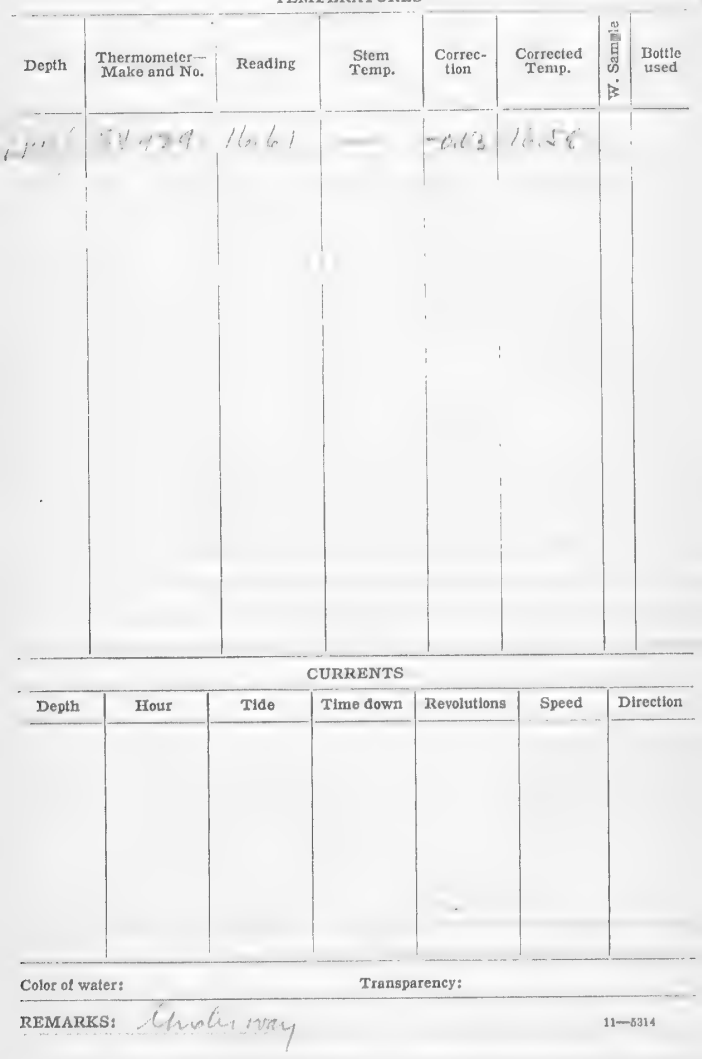

Force,

Sky: 
NETS, ITC., USTD

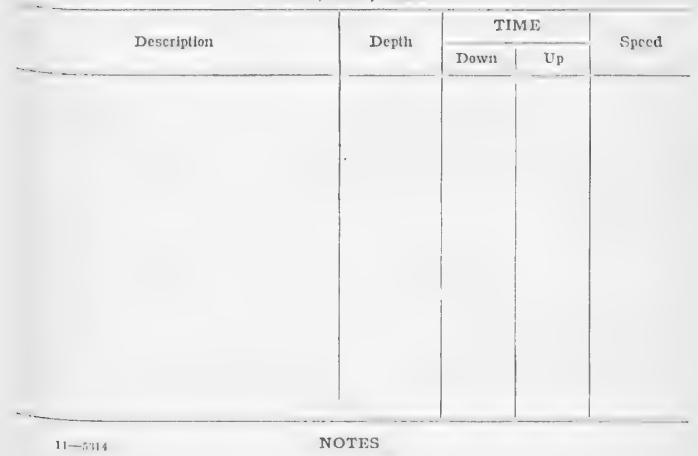




Station No. H $75 \%$ Date: $C<, 18 \%$

Hour: $9: 00$ A.M. to $\quad$. M.

Position: Lat. $30^{\circ} 45^{\prime} 5 J^{\prime \prime} N$ Long. $118^{\circ} 32^{\prime} 40^{\prime \prime} \mathrm{W}$ thut $* 6$. Chant 3000 tho

\section{Locality:}

Depth:

Bottom:

Sample:

Wind: Direction,

Force,

Sea:

Sky:

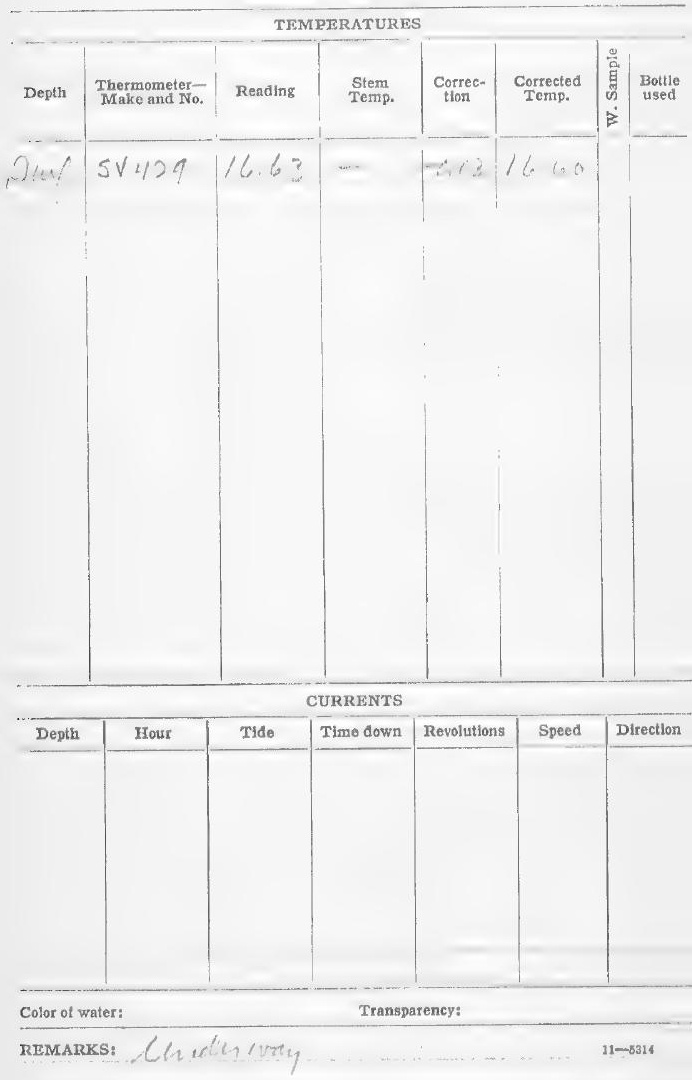


NETS, ETC., USED

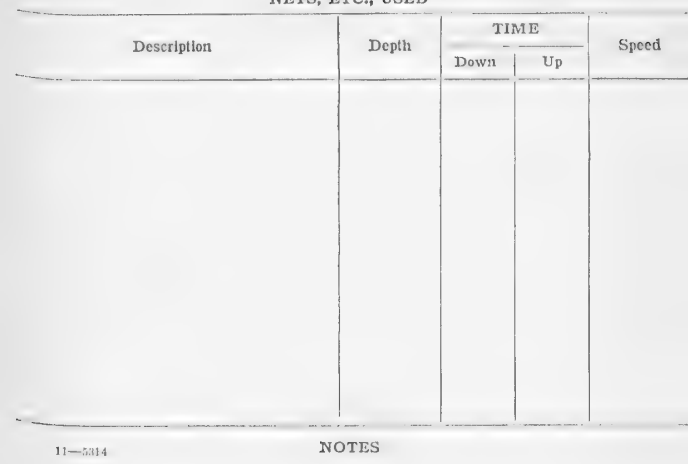


Position: Lat. $30^{\circ} 46^{\prime} 30^{\prime \prime} \mathrm{N}$ Long. $118^{\circ} 35^{\prime} 15^{\prime \prime} \mathrm{W}$ thut 6 chart 3000 H.

\section{Locality:}

\section{Depth:}

Bottom:

Force,

Wind: Direction,

Sea:

Sea
Sky:

TEMPERATURES

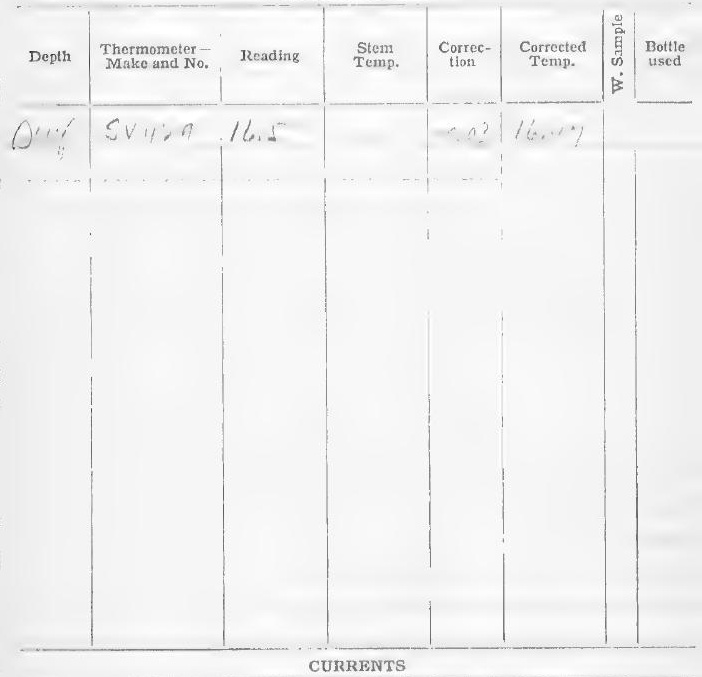

CURRFNTS

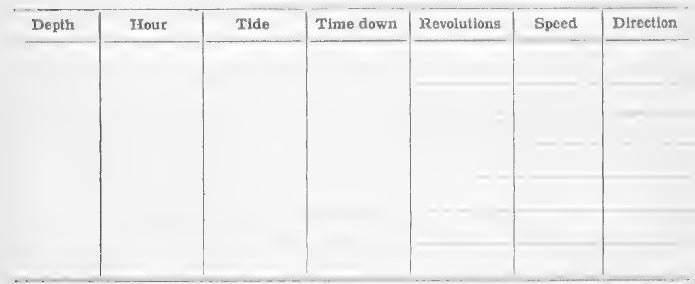

Sample: 
NETS, ETC., USED

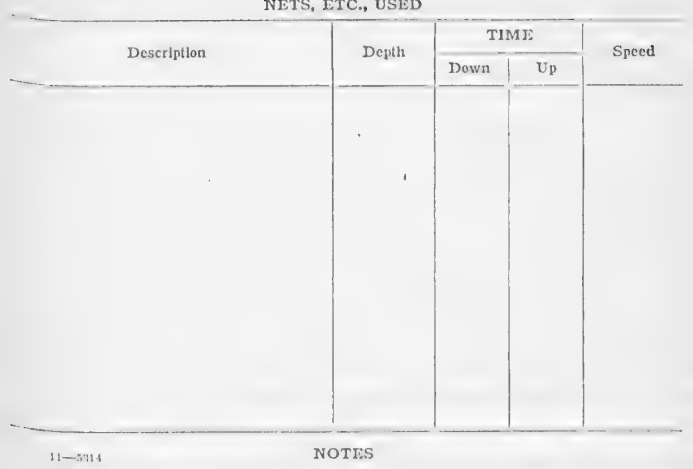


Station No. $F J \cap$ d Date: $r: 191$ . M.

Position: Lat. $30^{\circ} 47^{\prime} 0^{\prime \prime} \mathrm{N}$ Long. $118^{\circ} 38^{\prime} 0^{\prime \prime} \mathrm{W}$ Whit wh ching 3000 tho.

\section{Tocality:}

\section{Depth:}

Bottom:

Sample:

Wind: Direction,

Sea:
Sky:

Force,

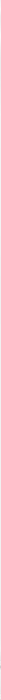

\begin{tabular}{|c|c|c|c|c|c|c|}
\hline Depth & Hour & Tide & Time down & Revolutions & Speed & Direction \\
\hline & & & & & & \\
\hline & & & & & & \\
\hline & & & & & & \\
\hline & & & & & & \\
\hline & & & & & & \\
\hline & & & & & & \\
\hline
\end{tabular}

Color of water:

Transparency:

REMARKS: Llumbin , vanty 11-3314 
NETS, ETC., USED

\begin{tabular}{l|l|l|l|}
\hline Description & Depth & Time & \\
\hline & & & \\
\hline & & & \\
\hline
\end{tabular}


Position: Lat. $30^{\circ} 47^{\prime} 50^{\prime \prime} N$ Long. $1 / 8^{\circ} 110^{\prime} 50^{\prime \prime} \mathrm{W}$ ohet th what 3000 ! 10

Locality:

Depth:

Bottom:

Sample:

Wind: Direction,

Force,

Sea:

Sky:

TIMPERATUTEES
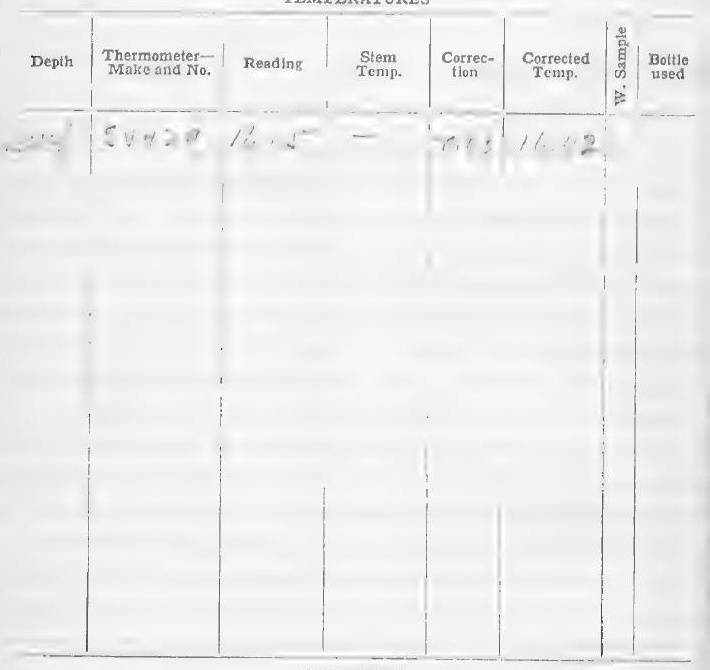

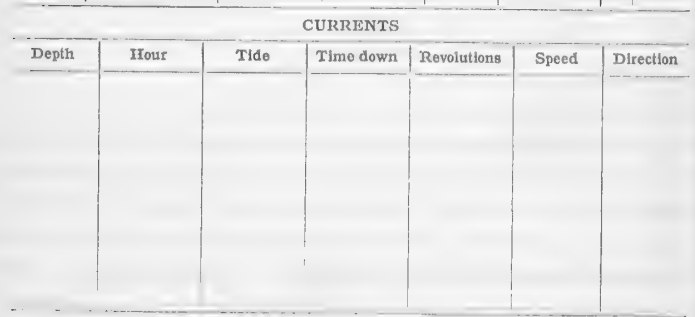

Color of water:

Transparency:

REMARKS: $t$ L w w w w w 
NITS, ETC., USED

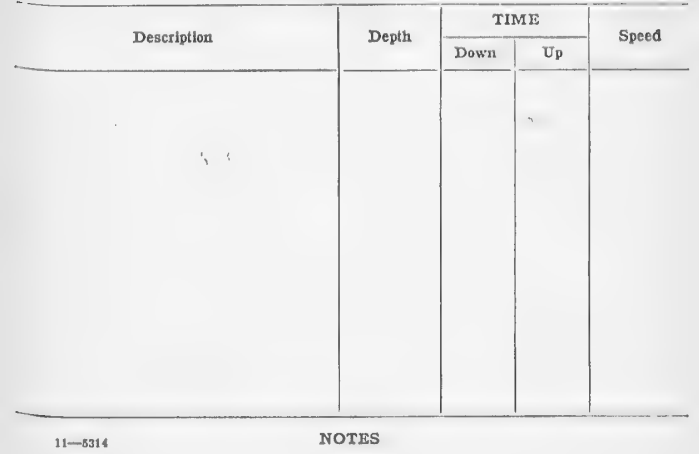


Position: Lat. $30^{\circ} 49^{\prime} 0^{\prime \prime} N$ Long. $118^{\circ} 46^{\prime} 0^{\prime \prime} \mathrm{W}$ ohut ${ }^{6}$. Chat 3000.17 .0

\section{Locality:}

\section{Depth:}

Bottom:

Sample:

Wind: Direction,

Sea:
Force,

Sky:

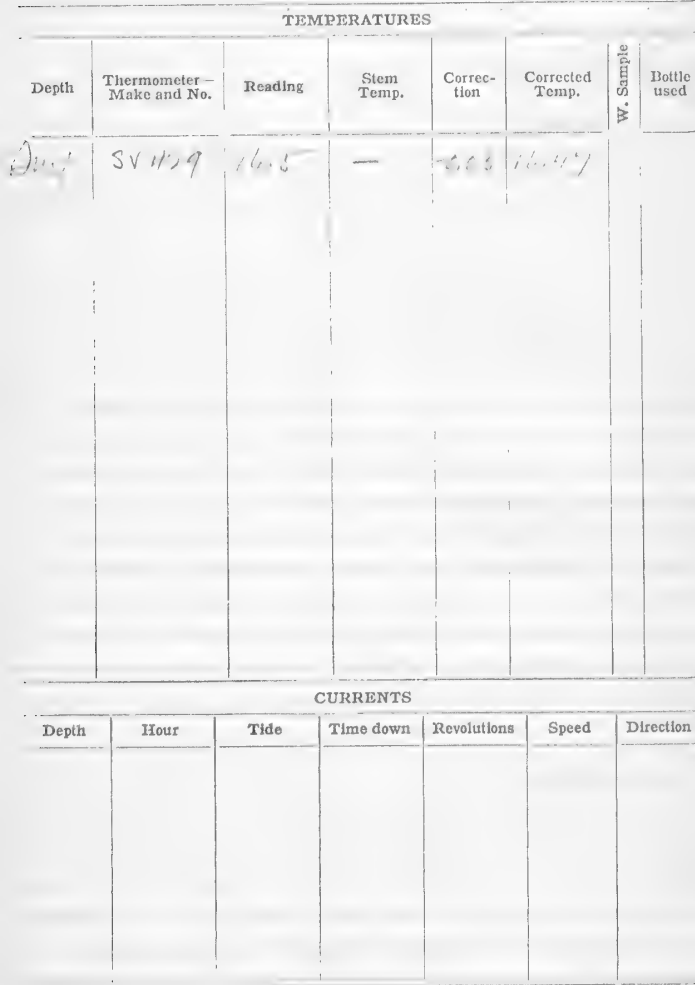


NETS, FTC., USED

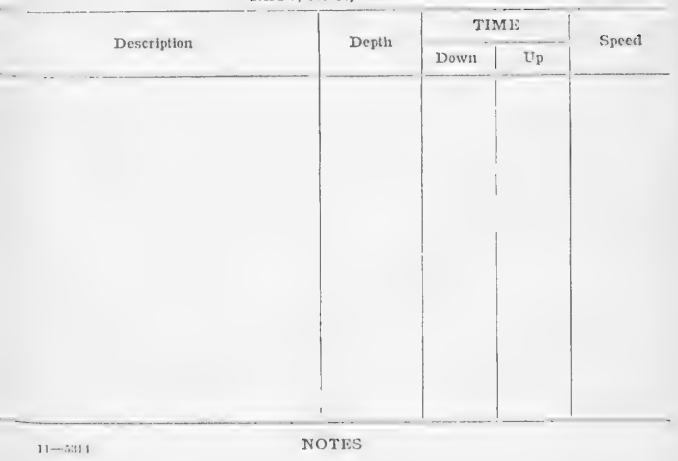


Hour: $12: 007$. M. to . M.

Position: Lat. $30^{\circ} 49^{\prime} 45^{\prime \prime} \mathrm{N}$ Long. $118^{\circ} 118^{\prime} 55^{\prime \prime} \mathrm{W}$ thent 286 chant $3000 \%$ t.

Locality:

Depth:

Wind: Direction,

Sea:

Sea:

TEMPTRATUIRES
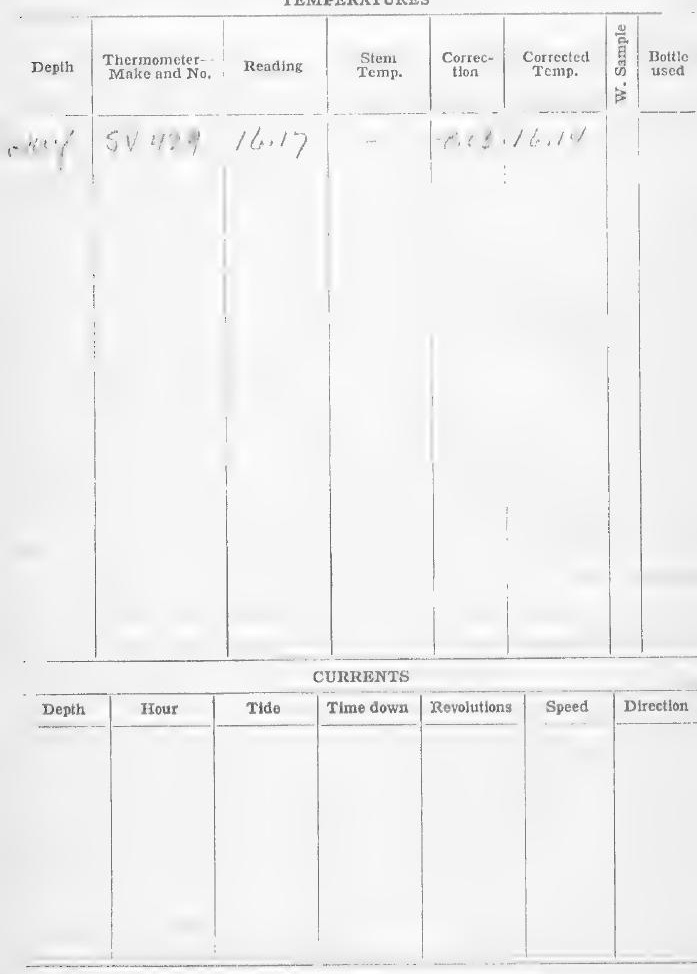

Color of water:

Transparency:

REMARKS: wlienter itrmy

$11-5314$ 
NETS, ETC., USED

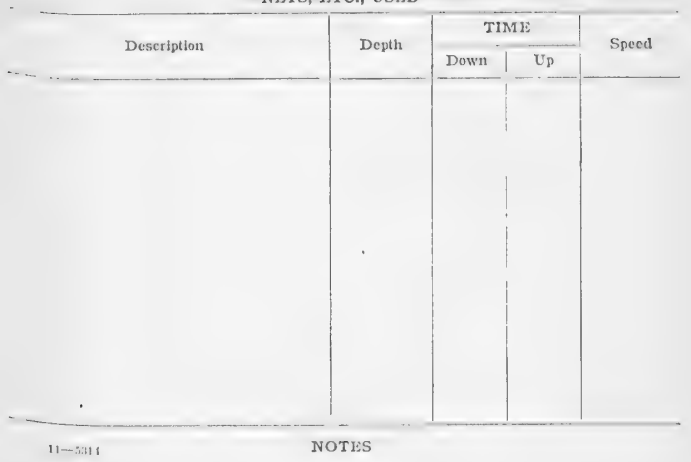


Position: Lat. $30^{\circ} 50^{\circ} 5^{\prime \prime} N$ Long. $118^{\circ} 5 L^{\prime} 0^{\prime \prime} \mathrm{W}$ oherit"6 chint sooutio

Locality:

Depth:

Bottom:

Sample:

Wind: Direction,

Sea:

\begin{tabular}{|c|c|c|c|c|c|c|c|}
\hline & & TF & RATU & & & & \\
\hline Depth & $\begin{array}{l}\text { Thermometer- } \\
\text { Make and No. }\end{array}$ & Reading & $\begin{array}{l}\text { Stcm } \\
\text { Temp. }\end{array}$ & Correc- & $\begin{array}{l}\text { Corrected } \\
\text { Tenı. }\end{array}$ & 㝵 & $\begin{array}{l}\text { Bottle } \\
\text { used }\end{array}$ \\
\hline$\because 11$ & $S \vee \% \because \because$ & 16.7 & & & 1 & & \\
\hline
\end{tabular}

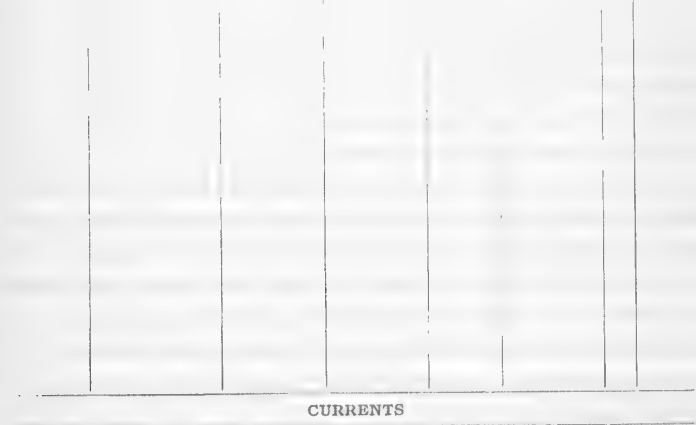

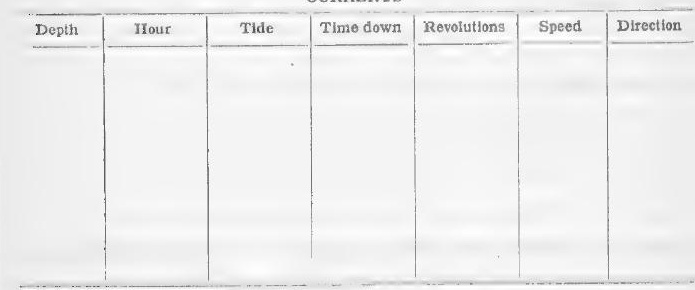

Color of water:

Transparency:

REMARKS: L/1 adis MR2, 
NETS, ETC., USED

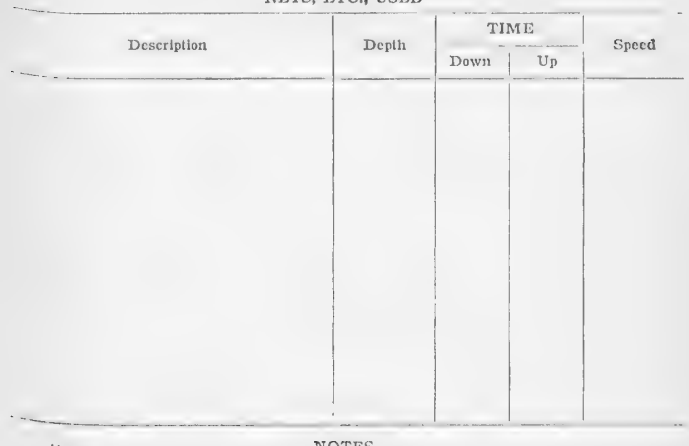



Hour:
$1: 00 P . M$. to
. M.

Position: Lat. $30^{\circ} 50^{\prime} 30^{\prime \prime} \mathrm{N}$ Long. $11.8^{\circ} 54^{\prime} 50^{\prime \prime} \mathrm{W}$ Dhut $\$$ " Chat $3000 \mathrm{H}$. O.

\section{Locality:}

Depth:

Bottom:

Sample:
Wind: Direction,

Sea:

TEMPIRATURES

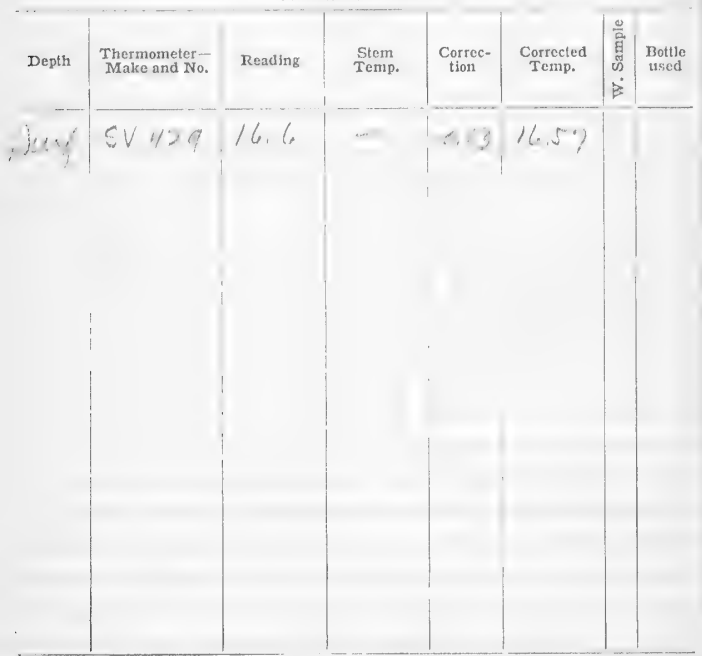

CURRENTS

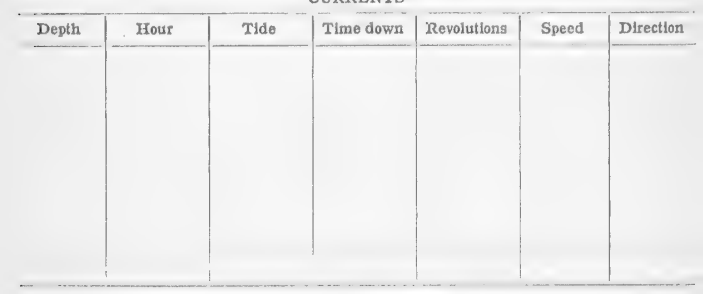

Color of water:

Transparency:

REMARKS: L Lu+elen: rifty

$11-8314$ 
NETS, ETC., USED

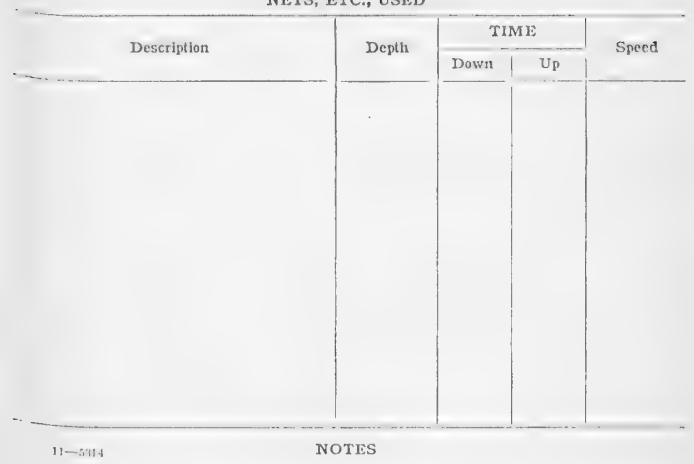


Station No. $\vdash / \% 5 / 1$

Hour: $/: 30$ P. M. to
Date:

$r^{*}, i, \cdots$

192

. M.

Position: Lat. $30^{\circ} 51^{\prime} \quad 0^{\prime \prime} N$ Long. $118^{\circ} 57^{\prime} 45^{\prime \prime} 11$ Aivit Chant $3000 \mathrm{H} .0$

Locality:

Depth:

Bottom:

Sample:

Wind: Direction,

Force,

Sea:

Sky:

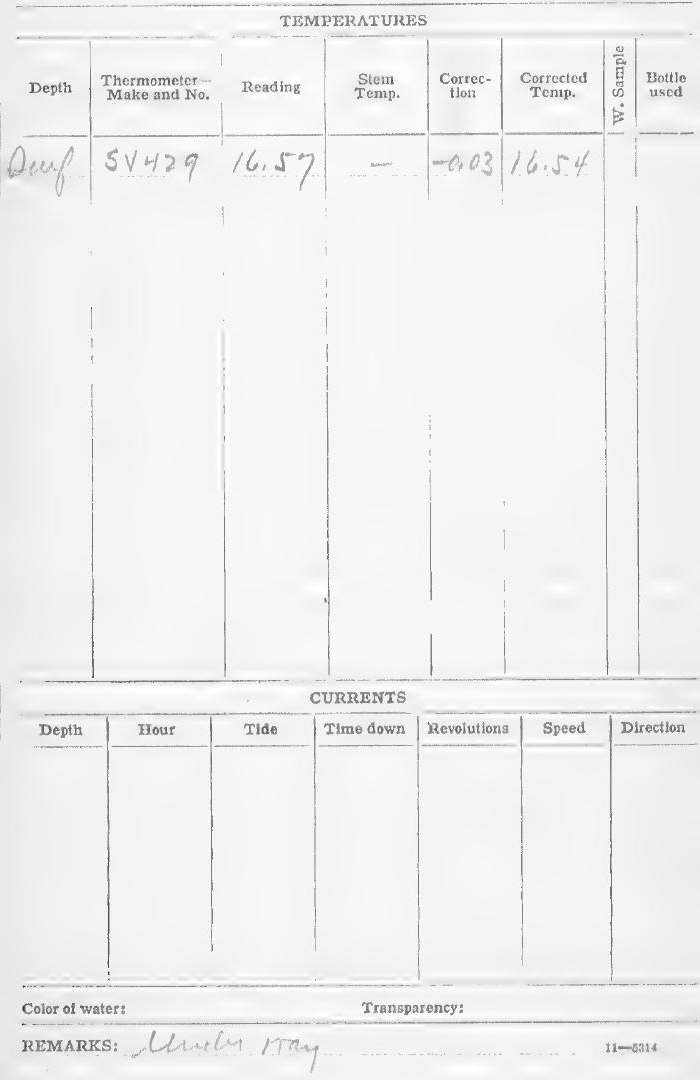


NETS, ETC., USED

Description


Hour: $2: 00 P . M$. to

. M.

Position: Lat. $30^{\circ} 51^{\prime} 30^{\prime \prime} \mathrm{N}$ Long. $119^{\circ} 0^{\prime} 50^{\prime \prime} \mathrm{V}$ Ohut 26 chant $3000 \mathrm{tt} 0$.

\section{Locality:}

Depth:

Bottom:

Sample:

Wind: Direction,

Force,

Sea:

Sky:

TEMPERATURES

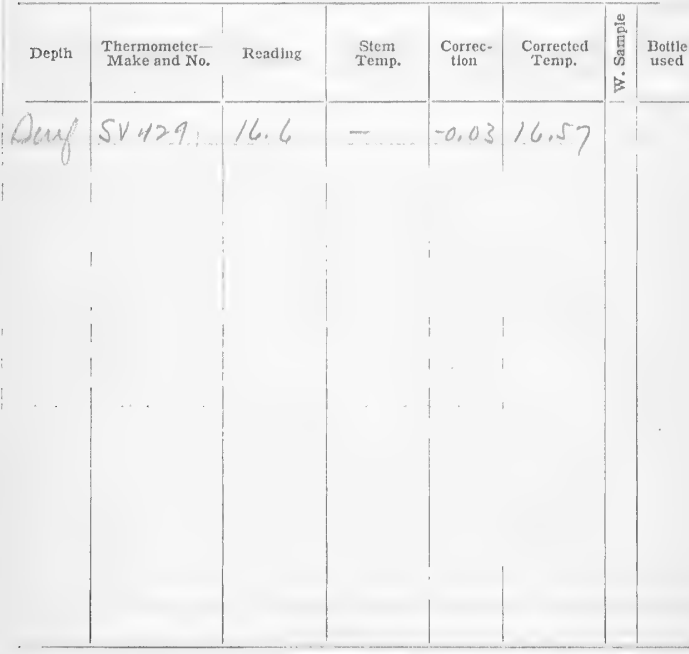

CURRENTS

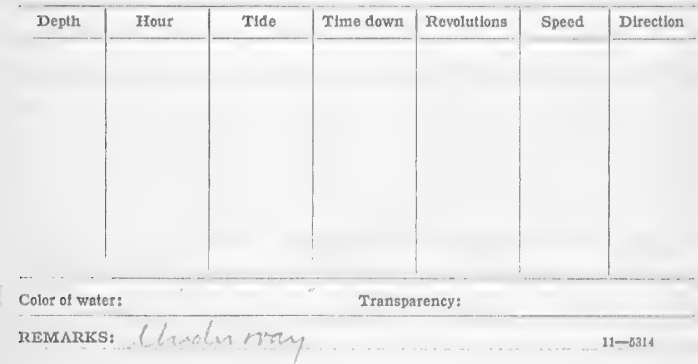


NETS, ETC., USED

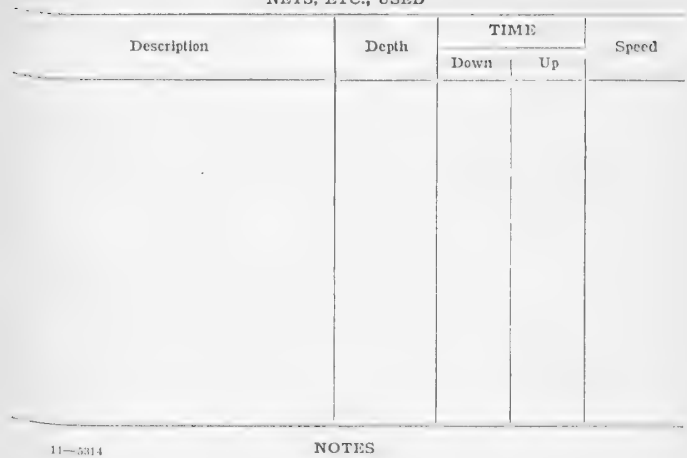


Position: Lat. $30^{\circ} \mathrm{r}^{\prime} 0^{\prime \prime} \mathrm{N}$ Long. $1 / 9^{\circ} 4^{\prime} 0^{\prime \prime} \mathrm{VV}$ ohert *6 L haid $=000 \% 6$.

Locality:

\section{Depth:}

Wind: Direction,

Sea:

Bottom:

Sample:

Force,

Slizy:

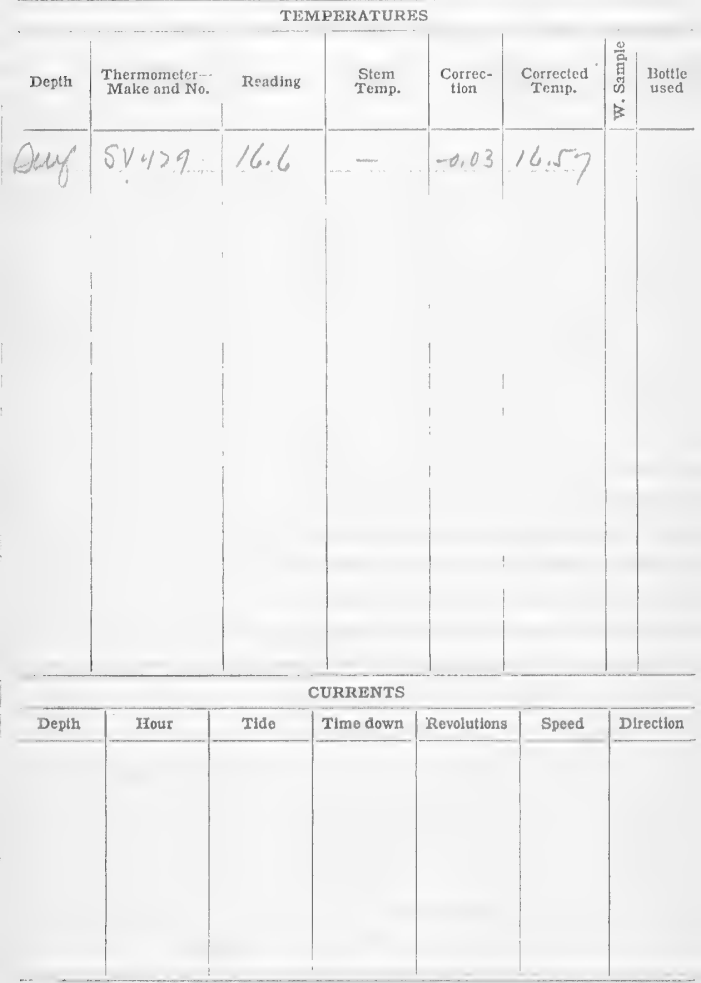

Color of water:

Transparency:

REMARKs: Lhaflut pray . 
NETS, ITC., USED

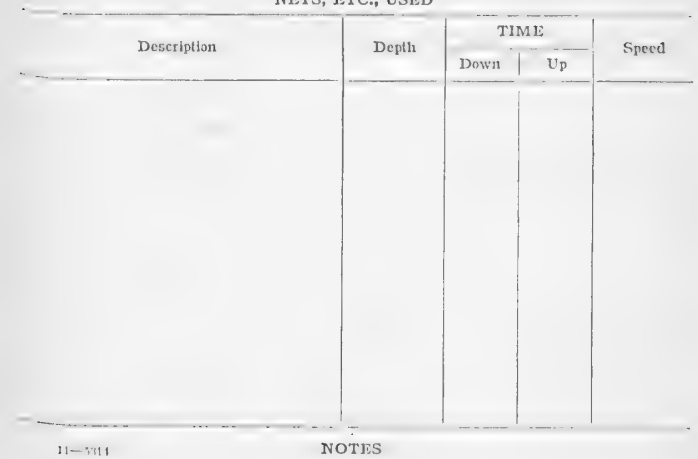


NETS, ETC., USED

Description


Hour: $3: 30, P$. M. to . M.

Position: Lat. $30^{\circ} 53^{\prime} 0^{\prime \prime} N$ Long. $119^{\circ} 9^{\prime} 30^{\prime \prime}$ L/ Whit *6 Chart $3000 \%$ t.

\section{Locality:}

Depth:

Force,

Siry:

Sea:

Wind: Direction,

Sample: $\ldots$

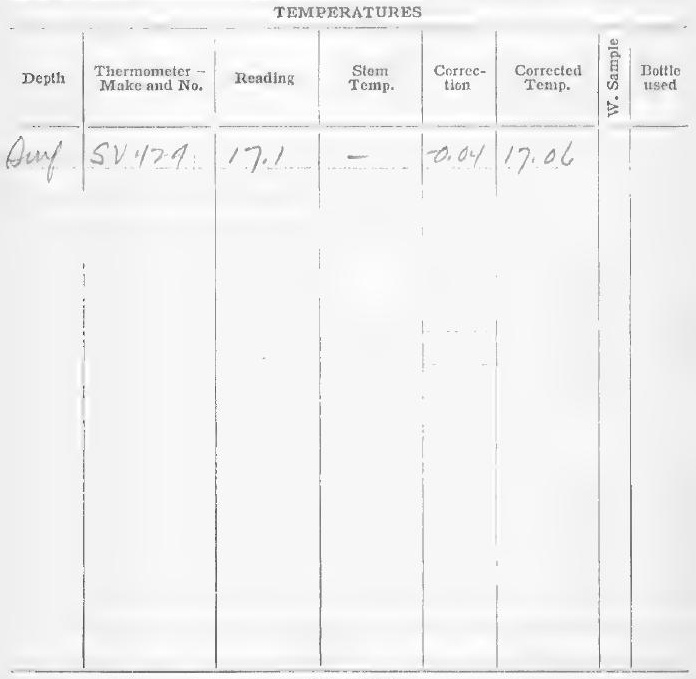

CURRENTS

\begin{tabular}{l|l|l|l|l|l|l} 
Depth & Hour & Tide & Time down & Revolutions & Speed & Direction \\
\hline & & & & & \\
& & & & & \\
\end{tabular}

Transparency:

develer rray 
NETS, ETC., USTD

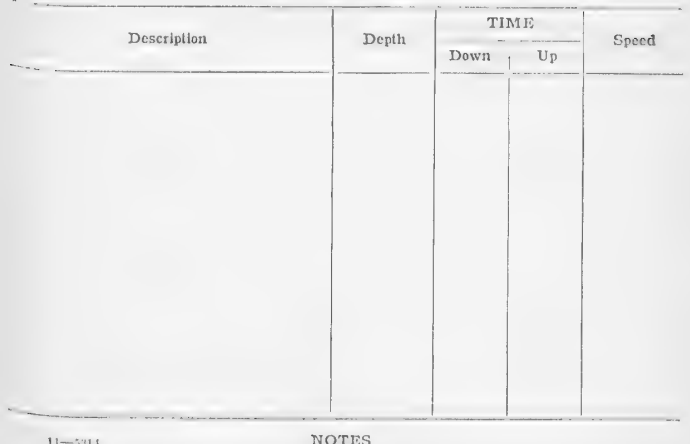

NOTES 
Date: QCF, 191\%

Hour: $7: 08$ A. M. to . M.

Position: Lat. $30^{\circ} 36^{\prime} 10^{\prime \prime} \mathrm{N}$ Long. $119^{\circ} 19^{\prime} 0^{\prime \prime} \mathrm{W}$ shut"6 Chart $3000 \mathrm{H} .0$.

Locality:
Depth:
Bottom:
Sample:

Wind: Direction,

Sea:

-

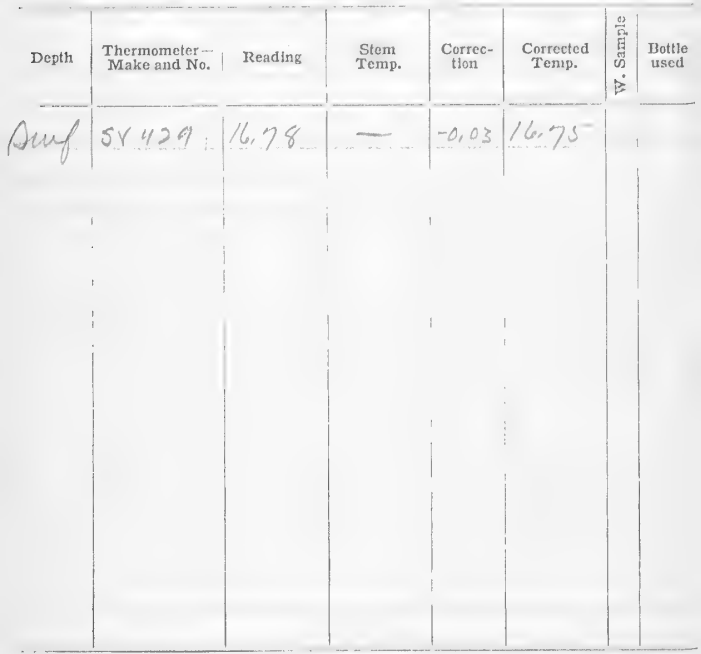

CURRENTS

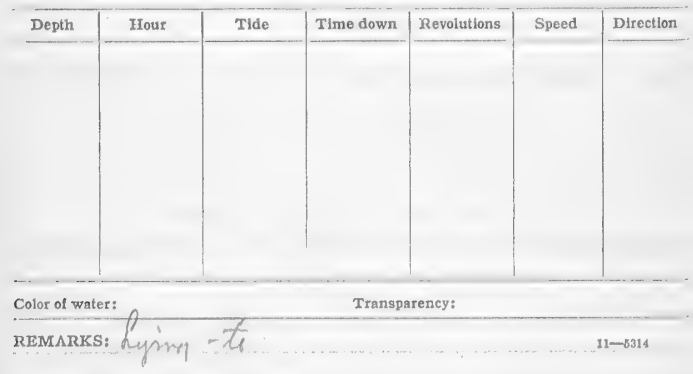


NETS, ETC., USED

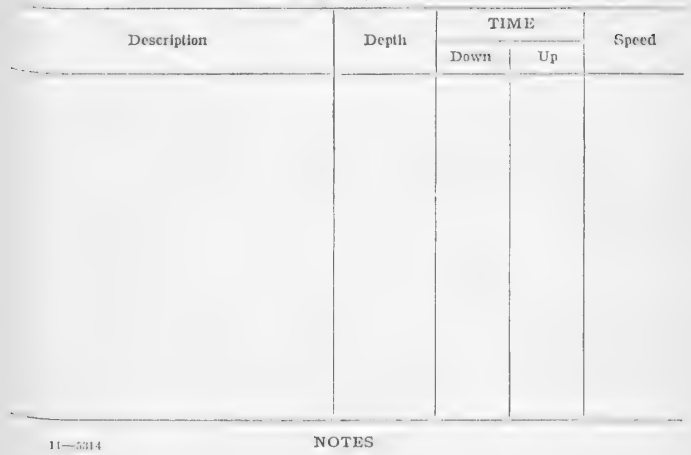




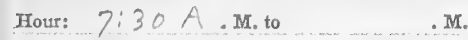

Position: Lat. $30^{\circ} 38^{\prime} S^{\prime \prime} N$ Long. $119^{\circ} 16^{\prime} 50^{\prime \prime} \mathrm{W}$ Ohut $* 6$ ehant $3000 \mathrm{H}, 0$.

Locality:
Depth:
Bottom:
Sample:

Wind: Direction,

Sea:
Force,

Sky:

TEMPERATURES

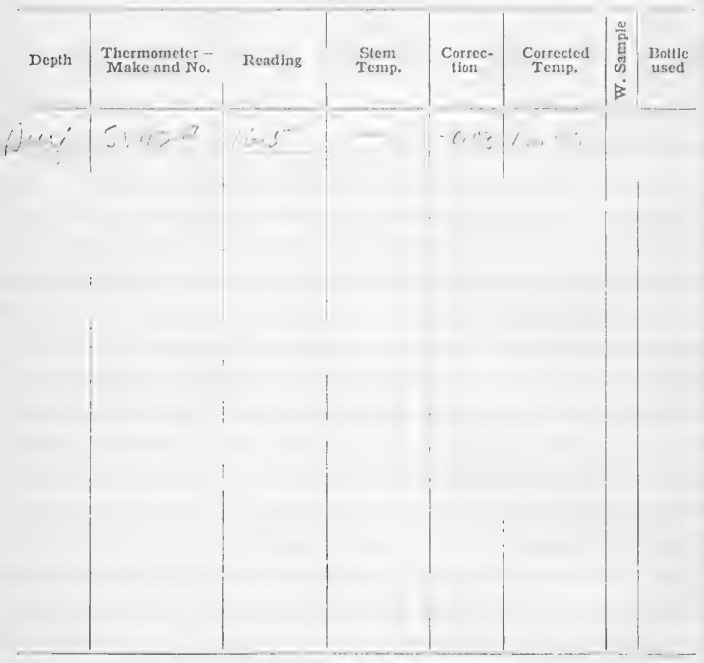

CURRENTS

\begin{tabular}{l|l|l|l|l|l|l}
\hline Depth & Hour & Tide & Time down & Revolutions & Speed & Direction \\
\hline & & & & & \\
& & & & & & \\
\end{tabular}

REMARKS: Llimlet intel 
NETS, ETC., USED

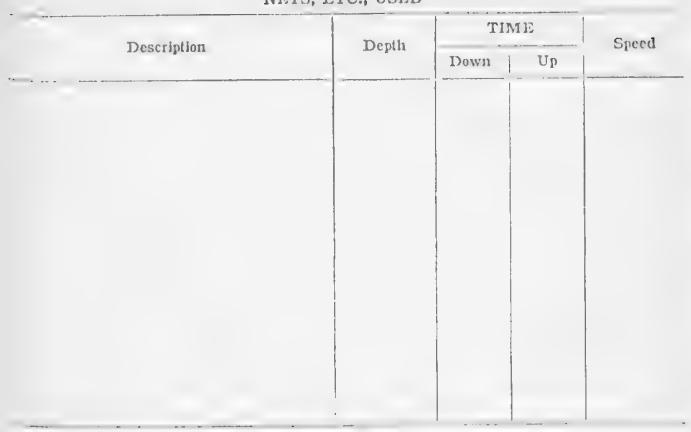


Station No. ". $q / \cdot$ Date: $<, \cdots, \% 1916$

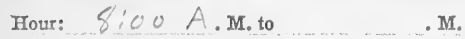

Position: Lat. $30^{\circ} 40^{\prime} 0^{\prime \prime} \mathrm{N}$ Long. $119^{\circ} 11^{\prime} 30^{\prime \prime} \mathrm{N}$

$$
\text { Dhut } 76 \text { Chant } 3000 \text { H.O. }
$$

Locality:

Depth:

Bottom:

Sample:

Wind: Direction,

Force,

Sea:

Sky:

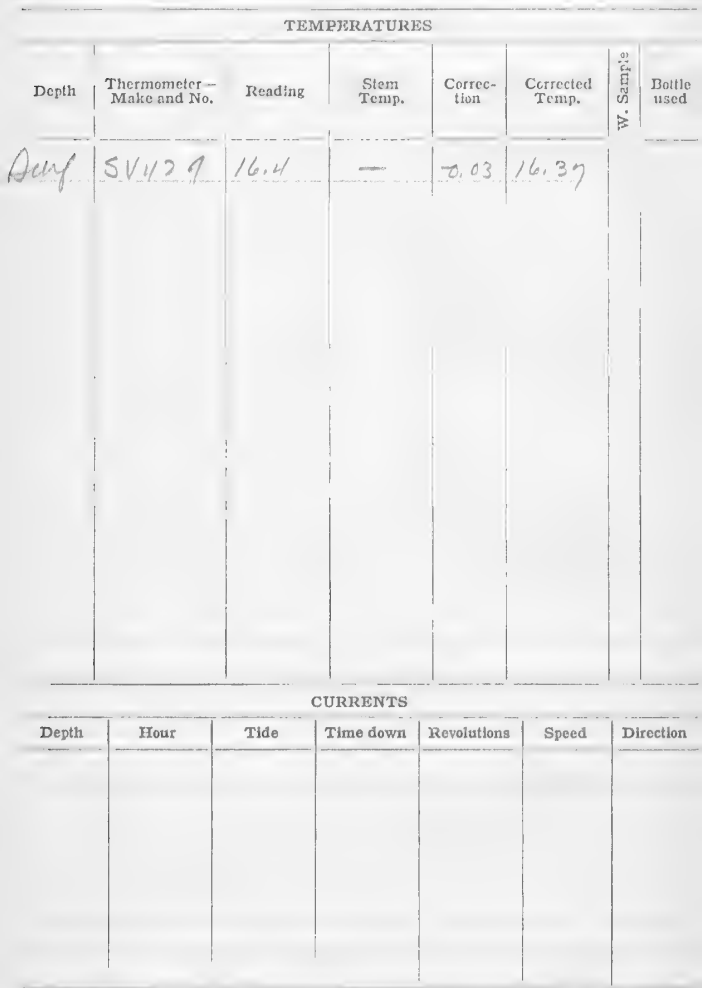

Color of water:

Transparency:

REMARKS: LAMCeI $1 \mathrm{my}$ 
NETS, ETC., USED

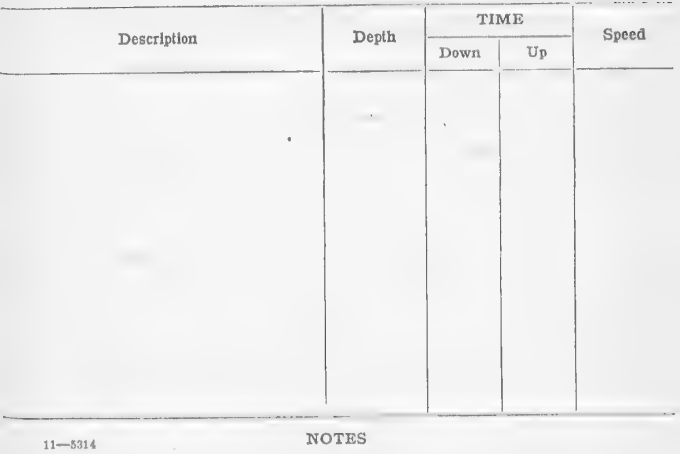


Date: $, \angle, \cdots, \cdots \quad 191$

Hour: $8: 30$ A. M. to

. M.

Position: Lat. $30^{\circ} 42^{\prime} 0^{\prime \prime} N$ Long. $119^{\circ} 1$, , $30^{\prime \prime} \mathrm{W}$ Dhut "6 Chant $3000 \mathrm{H} .0$.

Locality:

Depth:

Bottom:

Sample:

Wind: Direction,

Sea:
Force,

Sky:

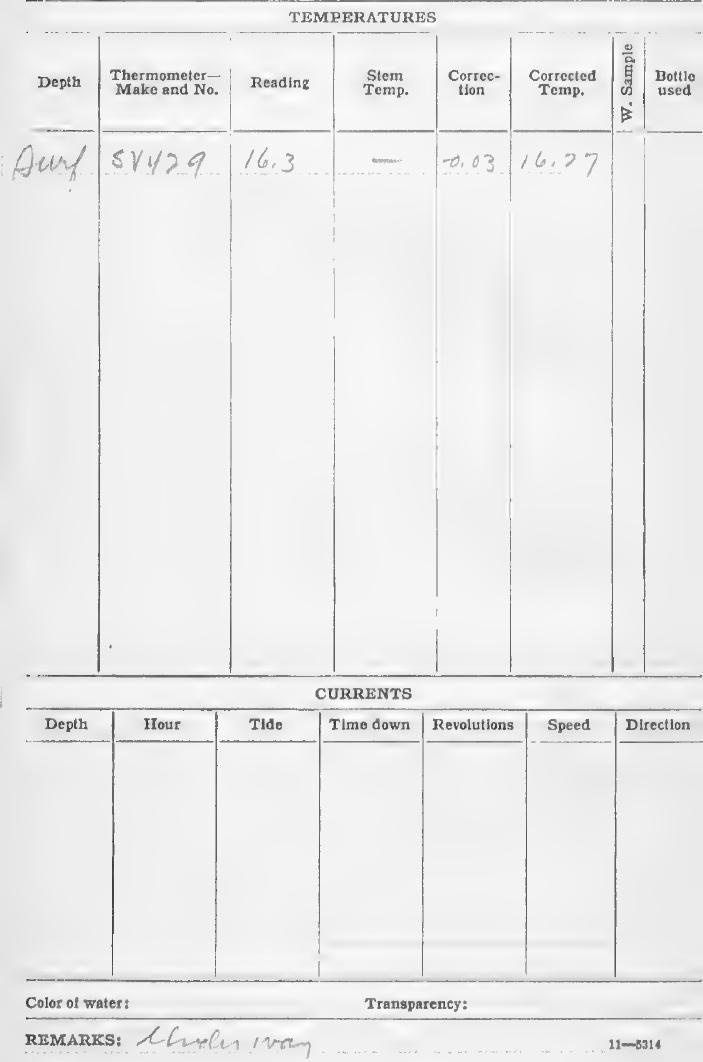


NITS, ETC., USED

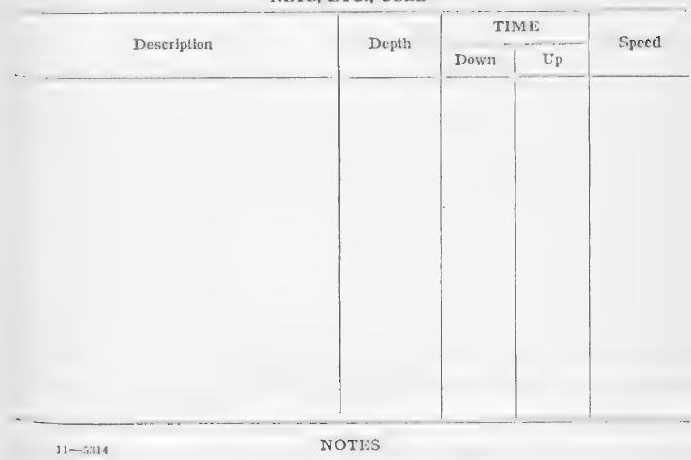


NITS, ITC., USED

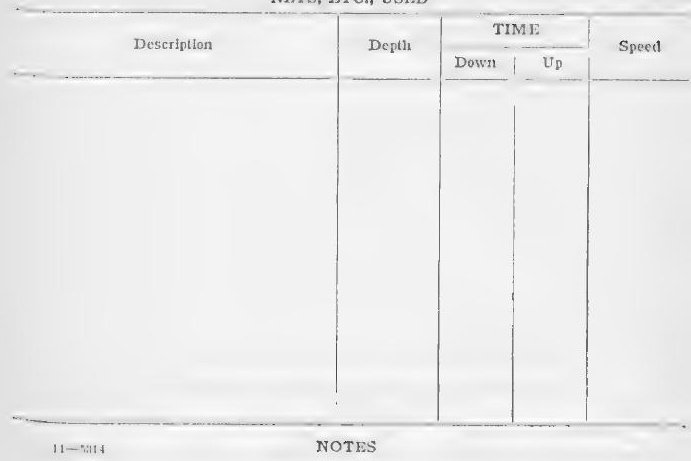


Station No. $t / 1,=:$

Date: $r, \cdot, \gamma$

$191<$

Hour: $9: 30$ A.M. to . M.

Position: Lat. $30^{\circ} 46^{\prime} 0^{\prime \prime} \mathrm{N}$ Long. $119^{\circ} \&^{\prime} 15^{\prime \prime} \mathrm{V}$ Dhont 6 Chart 3000 t. 0 .

Locality:

Depth:

Bottom:

Sample:

Wind: Direction,

Force,

Sea:

Slsy:

TEMPERATURJS

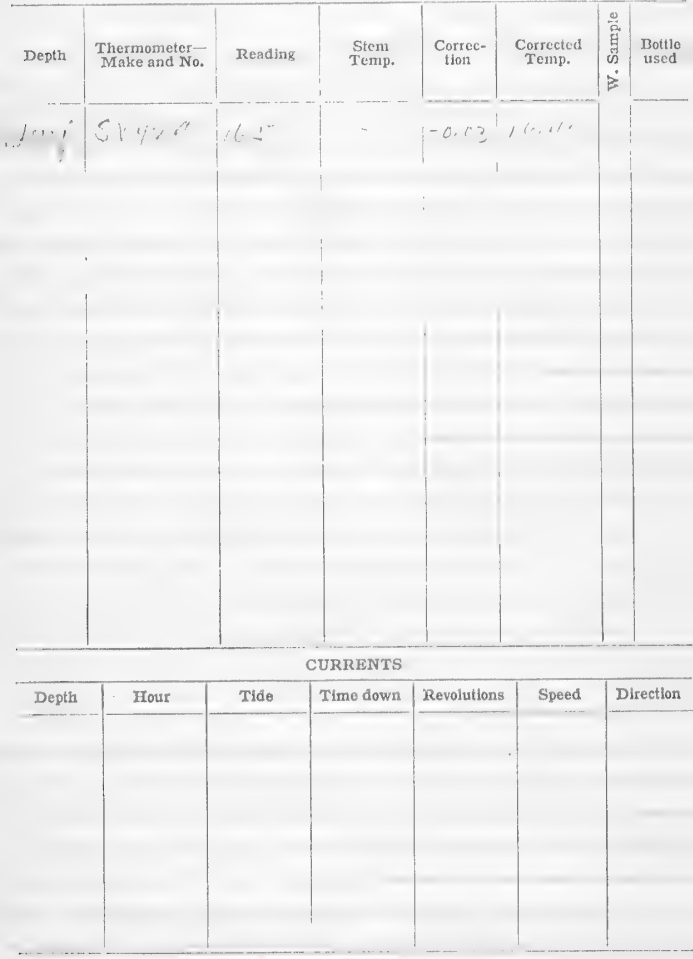

Color of water:

Transparency:

REMARKS:

Clumber may

$11-8314$ 
NETS, ETC., USFD

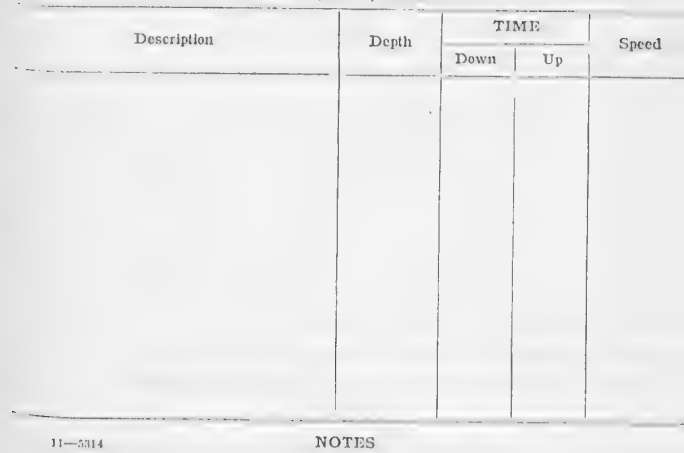


Position: Lat. $30^{\circ} 48^{\prime} 0^{\prime \prime} N$ Long. $119^{\circ} 6^{\prime}+0^{\prime \prime} 1 \mathrm{l}$ 1.) hut to ctant 3000 tt. 0 .

Locality:

Depth:

Bottom:

Sample:

Wind: Direction,

Force,

Sea:

Sky:

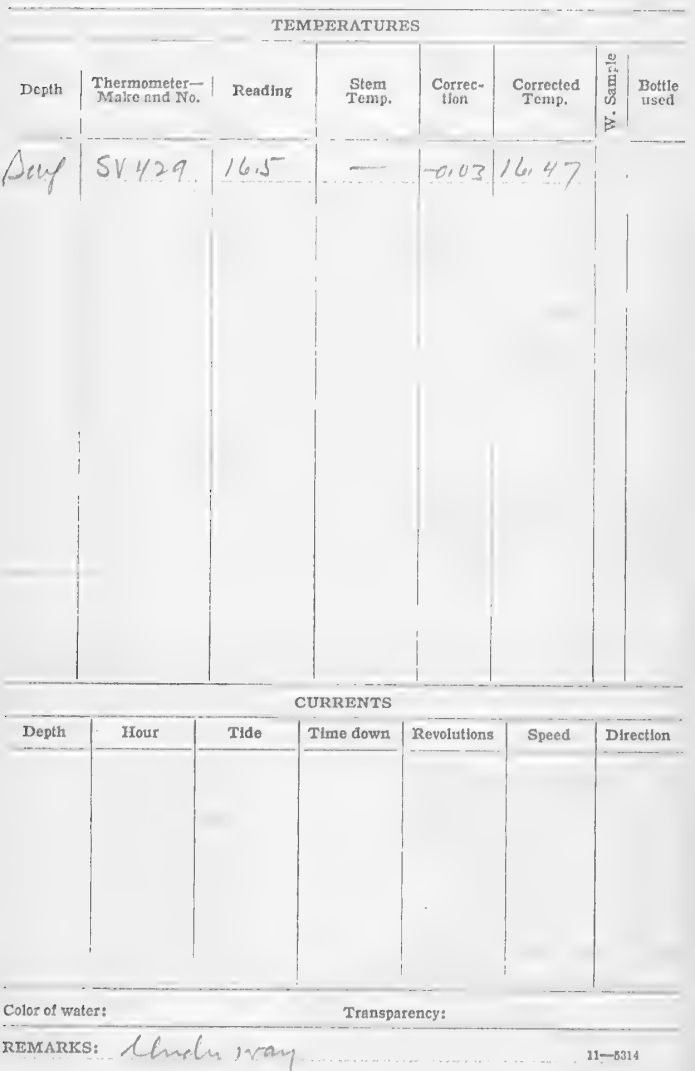


NETS, ETC., USID

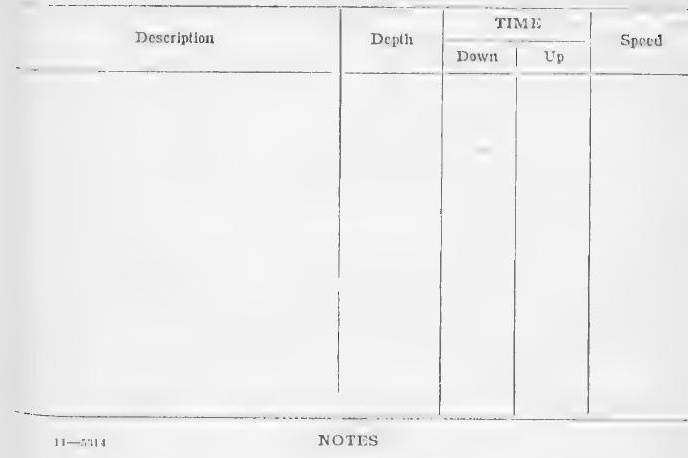


NETS, ITC., USFD

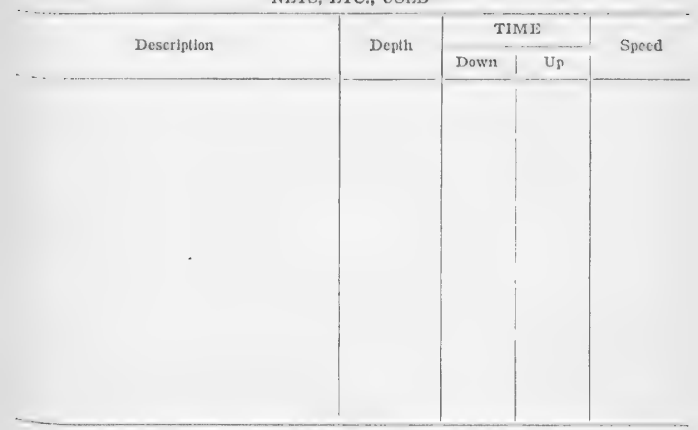

$11-5314$

NOTES 
NETS, ETC., USED

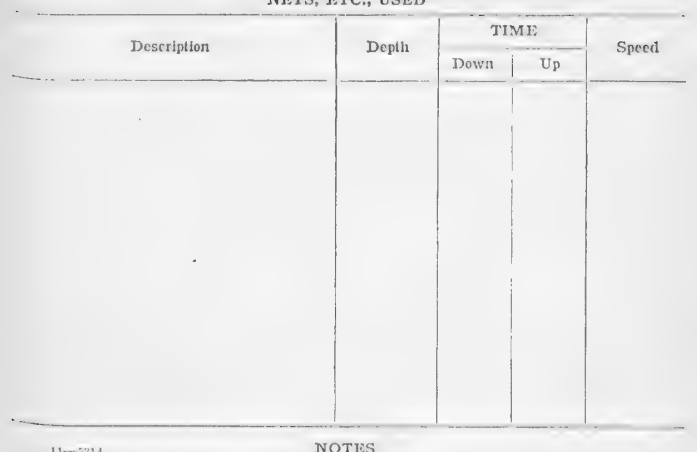

$11-\div 144$ 
NETS, ETC., USED

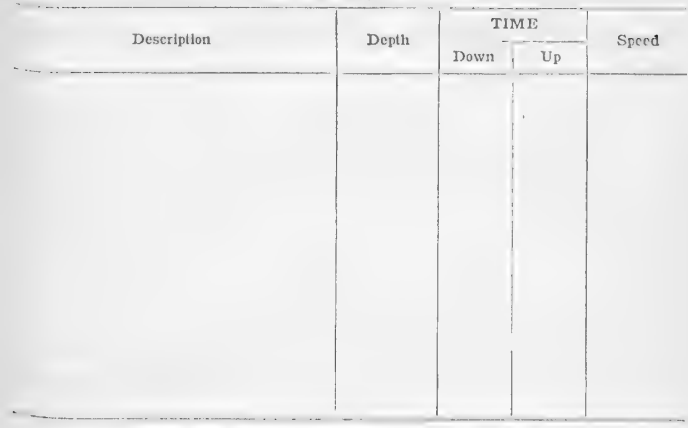

$11-2314$ NOTES 
Station No. $H 7527$

Hour: $1: 30 P$. M. to
Date: Out, 9

191 Position: Lat. $30^{\circ} 56^{\prime} 0^{\prime \prime} \mathrm{N}$ Long. $118^{\circ} 57^{\prime} 50^{\prime \prime} \mathrm{W}$
Ohut 6 Chant $3000+1.0$.

Locality:

Depth:

Bottom:

Sample:

Wind: Direction,

Force,

Sea:

Sky:

TEMPERATURES

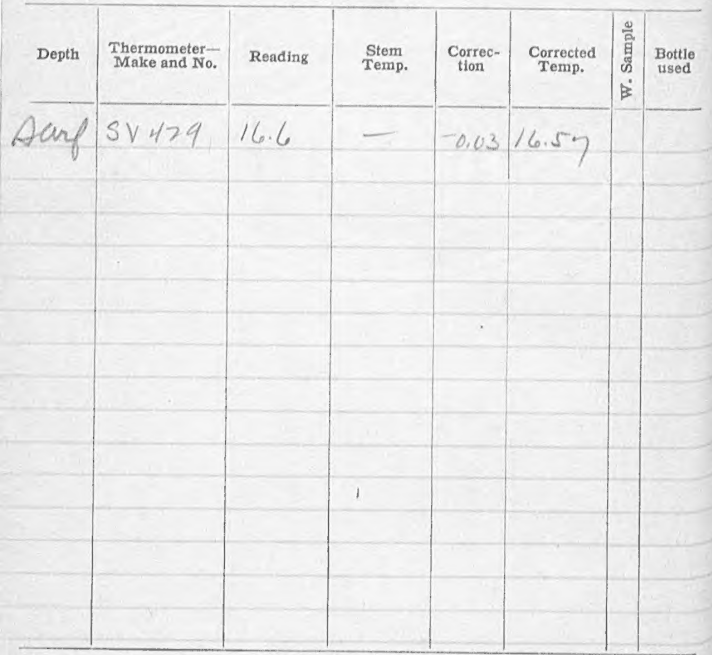

CURRENTS

\begin{tabular}{l|l|l|l|l|l|l}
\hline Depth & Hour & Tide & Time down & Revolutions & Speed & Direction \\
\hline & & & & & & \\
\hline & & & & & & \\
& & & & & & \\
& & & & & & \\
\hline
\end{tabular}

Color of water:

Transparency:

REMARKS:

choles may

. M.

ran 
NETS, ETC., USED

\begin{tabular}{l|l|l|l|l|l}
\hline Description & Depth & \multicolumn{2}{|c|}{ TIME } & \multirow{2}{*}{ Speed } \\
\hline & & Down & Up & \\
\hline & & & & & \\
\hline & & & & \\
\hline
\end{tabular}

$11-5314$

NOTES 
NETS, ETC., USED

\begin{tabular}{l|l|l|l|l|l}
\hline Description & Depth & Down & Up & Speed \\
\hline & & & & \\
\hline & & & & & \\
\hline & & & & & \\
\hline $11-5314$ & & & & & \\
\hline
\end{tabular}

